\begin{tabular}{|c|c|}
\hline $\begin{array}{l}\text { SpaceOps } 2010 \text { Abstract For } \\
\text { Do not extend beyond this one page. } \\
\text { Do not change font size (11). } \\
\text { Text-based symbols are OK, but embed } \\
\text { Graphics are not OK. } \\
\text { Read the Author's Kit for more details. }\end{array}$ & \multirow{2}{*}{$\begin{array}{l}\text { Tell us your presentation preferences: } \\
\text { Add only Y for Yes in the brackets [ ], N's are not needed. We encourage } \\
\text { flexibility - both oral and poster forums have their strengths. See website. } \\
\text { - I can present in either oral or poster sessions [ Y] } \\
\text { - I will only present in an Oral Session [ ] } \\
\text { - I will only present in a Poster Session [ ] } \\
\text { - I would like an ePoster Session because my topic suits that forum [ ] } \\
\text { - If selected as a poster presenter, I will consider a request to switch to } \\
\text { an oral presentation to cover for a withdrawn oral presenter [Y ] }\end{array}$} \\
\hline & \\
\hline \\
\hline \multicolumn{2}{|c|}{$\begin{array}{l}\text { Your Author list: (each author's name and affiliation) } \\
\text { David W. Scott - NASA MSFC (EO20) }\end{array}$} \\
\hline \multicolumn{2}{|c|}{$\begin{array}{l}\text { Your Abstract text: } \\
\text { Marshall Space Flight Center (MSFC) provides communication services for a number of real time environments, } \\
\text { including Space Shuttle Main Engine support and International Space Station (ISS) payload operations. In such settings, } \\
\text { control team members speak with each other via multiple voice circuits or "loops". Each loop has a particular purpose } \\
\text { and constituency, and users are assigned listen and/or talk capabilities for a given loop based on their role in fulfilling } \\
\text { the purpose. A "voice switch" is a given facility's hardware and software that supports such communication, and may } \\
\text { be interconnected with other facilities' switches to create a large network that, from an end user perspective, acts like a } \\
\text { single system. Since users typically monitor and/or respond to several voice loops concurrently for hours on end and } \\
\text { real time operations can be very dynamic and intense, it's vital that a control panel or "keyset" for interfacing with the } \\
\text { voice switch be a servant that reduces stress, not a master that adds it. Implementing the visual interface on a } \\
\text { computer screen provides tremendous flexibility and configurability, but there's a very real risk of over-complication. } \\
\text { (Remember how office automation made life "easier", which led to a deluge of documents that made life harder?) This } \\
\text { paper a) discusses some basic human factors considerations related to keysets, b) suggests what to standardize at the } \\
\text { facility level and what to leave to the user's preference, and c) provides screen shot mockups for a robust but } \\
\text { reasonably simple user experience. Concepts apply to keyset needs in almost any type of operations control or support } \\
\text { center. }\end{array}$} \\
\hline
\end{tabular}




\title{
Suggestions for Layout and Functional Behavior of Software-Based Voice Switch Keysets
}

\author{
David W. Scott ${ }^{*}$ \\ NASA - Marshall Space Flight Center, Huntsville, AL 35812
}

\begin{abstract}
Marshall Space Flight Center (MSFC) provides communication services for a number of real time environments, including Space Shuttle Propulsion support and International Space Station (ISS) payload operations. In such settings, control team members speak with each other via multiple voice circuits or "loops". Each loop has a particular purpose and constituency, and users are assigned listen and/or talk capabilities for a given loop based on their role in fulfilling the purpose. A "voice switch" is a given facility's hardware and software that supports such communication, and may be interconnected with other facilities' switches to create a large network that, from an end user perspective, acts like a single system. Since users typically monitor and/or respond to several voice loops concurrently for hours on end and real time operations can be very dynamic and intense, it's vital that a control panel or "keyset" for interfacing with the voice switch be a servant that reduces stress, not a master that adds it. Implementing the visual interface on a computer screen provides tremendous flexibility and configurability, but there's a very real risk of overcomplication. (Remember how office automation made life "easier", which led to a deluge of documents that made life harder?) This paper a) discusses some basic human factors considerations related to keysets implemented as application software windows, b) suggests what to standardize at the facility level and what to leave to the user's preference, and c) provides screen shot mockups for a robust but reasonably simple user experience. Concepts apply to keyset needs in almost any type of operations control or support center.
\end{abstract}

\section{Introduction}

$\mathrm{F}$ or several years, MSFC has used Voice over Internet Protocol (VoIP) to support remote user connections to its mission operations voice switch. To the author's knowledge, this was one of the earliest applications of VoIP in an operational setting. As VoIP system costs decreased and reliability improved, Marshall decided to adopt that approach for the main voice switch as well. Software-based keysets were desired due to multiple types of flexibility - appearance and function can be readily changed, and they can be hosted on dedicated hardware or run in a software window on multipurpose computers. In 2009, MSFC set up a demonstration system (hereafter called the Demo System) for several months and invited console operators and technicians to exercise it and provide comments. Some "keysets" were implemented as application windows on multipurpose computers, while others were hosted on dedicated touch-screen devices. This paper is based on the author's response to that demonstration

\section{Fundamental Principles for Interface Suggestions}

The suggestions made in this paper are based on the following principles:

- Emphasize visual simplicity. "Less is more." (Most users do not need insight into voice switch details.)

- Symbolic colors should be set group-wide. Client should appear identical across operating systems.

- Use high target value (bright colors and/or high contrast) sparingly, and only for critical information. Use strong yellow and red only for cautions and warnings, respectively.

- Color is supplemental, not primary. The interface should be intuitive even for a colorblind operator.

- Exploit the power of individual logons: give users appropriate control of a) what info to show or hide and b) certain visual interface behaviors. Items of great interest to communications technicians can be distracting to operations personnel.

- Make command key behavior consistent.

\footnotetext{
* Computer Engineer, Ground Systems Development and Integration Branch, NASA EO50, Marshall Space Flight Center, Huntsville, AL 35812
} 
- To the greatest extent possible,

$\circ$ Keep basic voice switch functions operational even during configuration processes

o Do not cover the main key matrix with other windows or data

o Do not resize the main window.

\section{Screen Images, Mockups, and Notes}

This section is essentially a series of screen images and/or mockups with notes on the following topics. (Except as noted, topics refer to the suggested interface.)

- Demo System Overview

- Legacy Keysets

- Suggested Interface

- The Options Menu

- The Mute Function

- Demo System Volume Configuration and Loop Assignments

- Volume-Setting Scheme

- Assigning Voice Loops to Matrix Keys

- User-Configured Interface Behavior

- Telephone Interface

- User-Controlled Phone Patch

- Vertical Panel Options for the Demo System and the Suggested Interface

Within the mockups and notes:

- Screen window mockups have the same 16:9 aspect ratio as the dedicated touch-screens used in the evaluation. (Presumably, application window keysets would use the same ratio.)

- Text annotations on mockups are in [ red type with brackets ].

- The terms key, button, or tile are used interchangeably.

- Select, press, or push means physically pressing a key via touch screen or clicking it with a mouse. 


\section{A. Demo System Overview}

Figure 1 provides an overview of the Demo System.

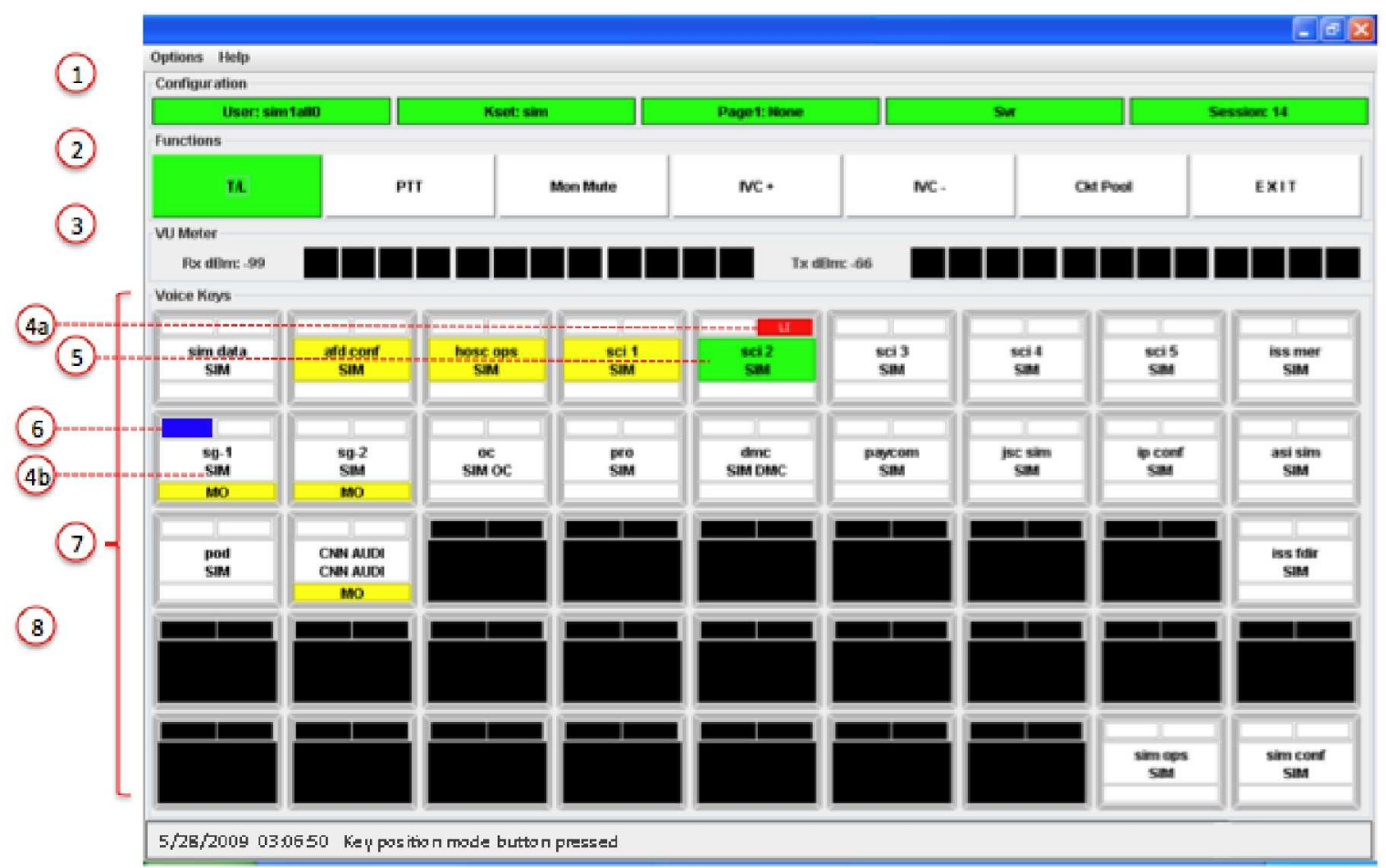

Figure 1 - Basic Features of the Demo System

NOTE - The numbered paragraphs below correspond to the numbered circles in Figure 1, and indicate features the author sought to improve upon in the Suggested Interface.

1. The information on the Configuration line is not terribly important to an operator trying to manage voice loops, so there is no need to have it in bright green at the top of the display. (Heartbeat information becomes important if a heartbeat anomaly occurs, but exception monitoring could be used to set an appropriate high value color.)

2. The Function keys work as follows:

- "Key-Press Behavior" - Changes how the system responds when an individual loop button in the 9x5 matrix is "pushed" (touched on touch-screen or clicked on with a mouse) repeatedly.

Mon - Key toggles between Monitor and Off.

$\mathrm{T} / \mathrm{L}$ - Key toggles between Talk/Listen and Off.

Cycle - Key cycles from Monitor to Talk/Listen to Off.

In T/L or Cycle, a loop in Talk/Listen goes to Monitor if user selects a different loop for Talk/Listen.

Communications technicians sometimes switch among behaviors, but most non-technical users will settle on a single setting.

- PTT (Push-To-Talk) - When pushed, transmits the user's voice if a loop is selected for "Talk". Changes color whenever set is "keyed", whether via this tile or by a PTT switch on the user's headset or microphone.

- Mon/Mute - When pressed, all loops selected for listening except for the current "Talk" loop are silenced, but remain lit. A second press on Mon/Mute makes them audible again.

- IVC+ and IVC- (Individual Volume Control + or -) - Raise and lower the relative volume of a selected loop. (Global volume settings for headseats and/or speakers are accessed via the Options menu.)

- Ckt Pool - Opens a screen for assigning voice loops to specific keys in the matrix.

- Exit - Shuts down the keyset software. 
Most of these functions are not used with great frequency.

3. The VU meters seem much larger than needed. (Once a keyset is set up to an operator's satisfaction, volume levels will be in an acceptable range.) Large meters could detract from the main job of managing communications.

4a. Link status (LI, LE for Link Initiated and Link Established) appears on a given loop key only if user is authorized for Talk/Listen on that loop.

4b. Link class (MO, RTC, etc. for Monitor-Only, Restricted Talk Capability, etc.) appears only if loop is not authorized for TLM (Talk/Listen/Monitor).

NOTE - The values for link status are of interest to communication technicians, but may distract non-techies, especially if given high target value. The typical operator just wants to know which loops they are hearing and which one they will talk on when they activate PTT.

5. The single most important thing on this display right now is that this is the loop the operator will talk on if they key up - signified by green. Having so many other bright areas on screen detracts from this, plus can cause eye strain after hours of viewing. A colorblind user might not be able to distinguish this from the yellow associated with a monitored loop. Also, the borders and shading on the keys use real estate and add clutter.

6. Solid blue appears when there is traffic on the loop.

7. Since the primary purpose of the display is to access voice loops, the author believes this whole block should be at the top of the page.

8. The large expanse of black creates high target value that detracts from the important information, i.e., status of assigned loops. 


\section{B. Legacy Keysets}

For the better part of 30 years, MSFC flight controllers have used the Light Emitting Diode (LED) and membrane-based keyset hardware shown in Figure 2.

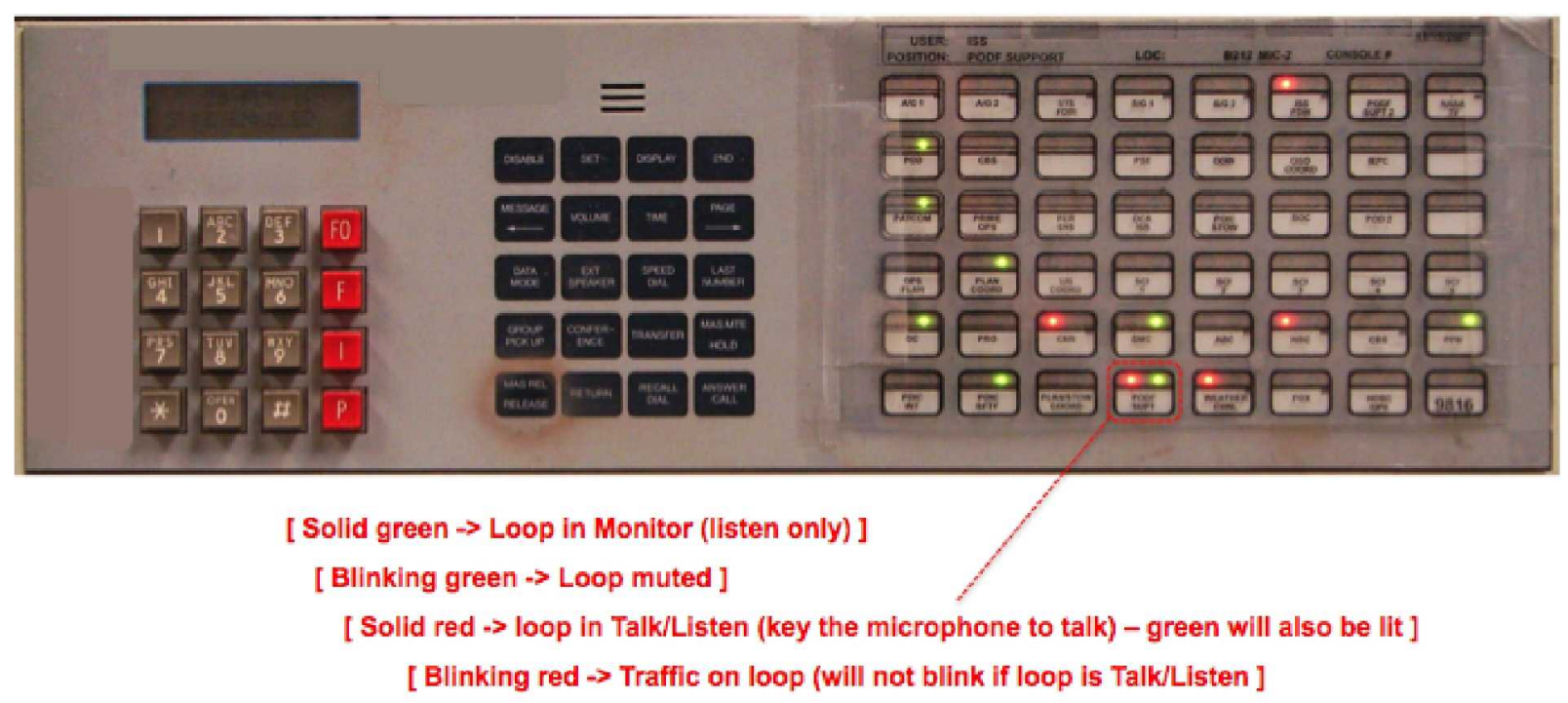

\section{Figure 2 - LED and Membrane-Based Keyset}

Loop key behavior as programmed for MSFC was to cycle between Talk/Listen (if authorized for that loop) and Monitor. To turn a loop off, a user had to press the Release Key (lower left key in the group of command buttons between the touchtone keypad and the loop matrix) followed by the desired key. The entire matrix could be cleared by pressing the " 2 nd" key (analogous to a typewriter Shift key) in the upper right, then the Release key.

Command key behavior was very consistent. In almost all cases, user pressed a command key (and sometimes a $2^{\text {nd }}$ command key modifying the action of the first one), then the key for the loop to be affected.

\section{Suggested Interface}

Figure 3 provides an overview of a suggested software-based interface inspired by the legacy hardware. 


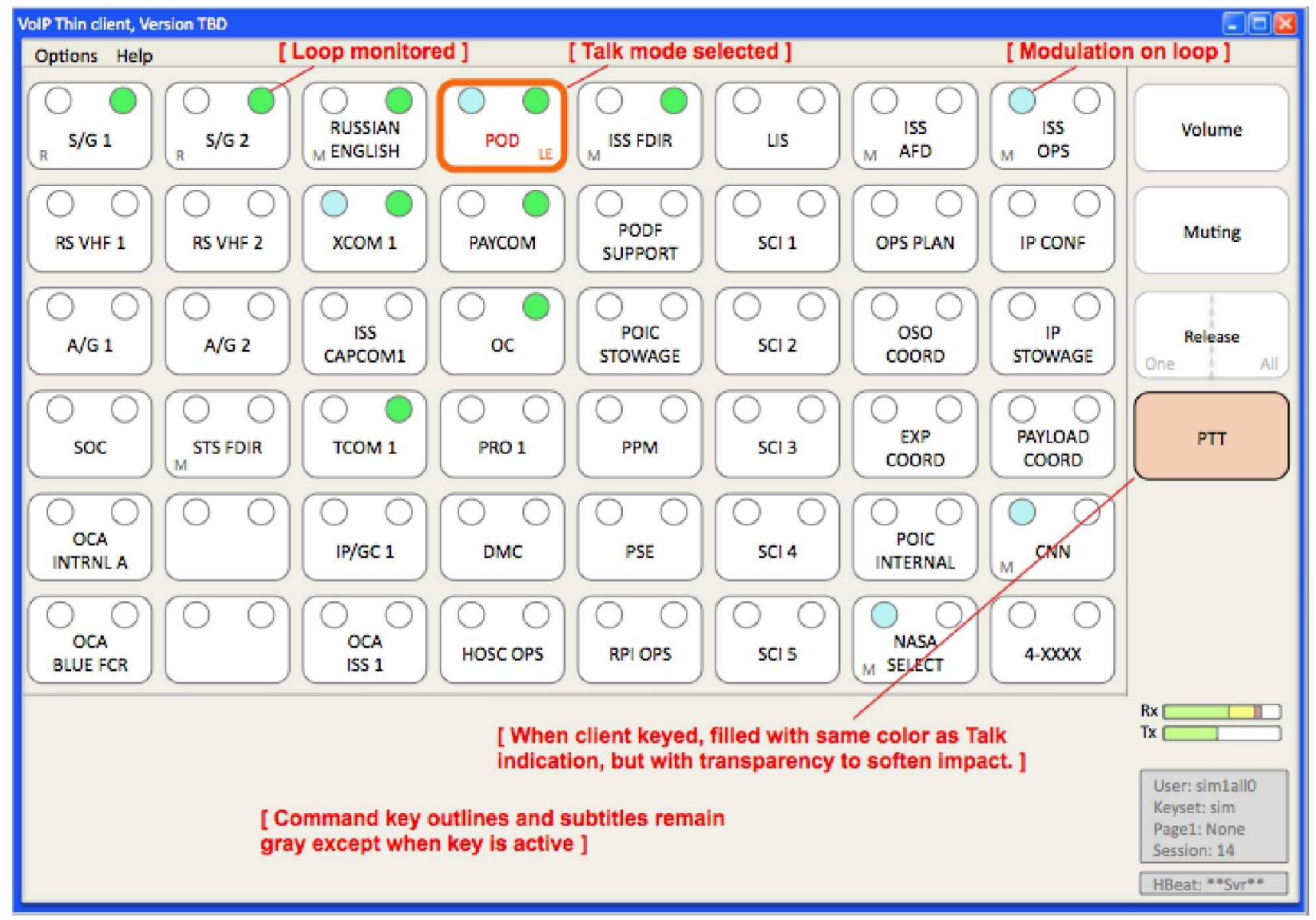

Figure 3 - Basic Features of the Suggested Interface

Highlights of the suggested interface are:

The rounded edges of the basic keys are easier on the eyes than hard-edged rectangles.

The small circles provide enough indicator real estate without overpowering.

The following features de-emphasize ancillary data so that important information stands out:

- Gray outlining of keys and indicator circles. No shadows.

- Light gray dotted lines and sub-text on Release key.

- Background color not grey (avoid monotony), but very muted.

- Ancillary data downsized and moved to lower right corner

- VU meter (optional display) outlined in gray and uses pastel colors

- ID data (optional display) in gray

- Heartbeat (always displayed) normally in gray, changes to yellow/red if out of limits

The author chose the 6x8 matrix over the 9x5 used in the Demo System because

- It leaves more screen real estate at the bottom of the page so that control menus (shown in Figures 7, 9, 10, and 11) do not block the main key matrix.

- The author preferred having 3 more loops (benevolent greed).

- Smaller aspect ratio makes it easier to navigate the matrix.

The functions of the Volume and Muting keys are explained in text accompanying Figures 7 and 5, respectively.

The functions of the Release key are:

- Pressing the left side of the Release key, then the desired matrix key, turns the associated loop Off.

- Pressing the right side of the Release key results in a dialog:

Do you really want to release ALL circuits? YES NO

"YES" turns all loops in the matrix Off, including one selected for Talk. "NO" cancels the dialog. 
Indications for Loop Class and Link Status are optional (see Figure 9) because they are of low interest for the typical non-techie user, but their behavior when shown is:

- Loop Class, e.g., TLM, MO, RTC - lower left corner of loop key in small type and grayed.

- Link Status, e.g., LI, LE - lower right corner of key in small type, same color as Talk indicator.

- Mnemonic values should be defined in system tables. Suggest blank for TLM, M for Monitor Only, R for RTC, but this should be a matter of local preference

NOTE - Some other drawings in this paper show smaller keys. The author believes this drawing has a very effective balance of key size versus space between keys, especially for touch screen use, but further experimentation involving multiple operators would probably be a good thing.

NOTE - The author may be a bit biased towards these shapes because they have been in front of him for many years. However, he has noticed that traffic light lenses are almost universally round and most road signs (and even rectangular traffic signal housings) have rounded edges. Since keysets help us control and navigate voice traffic, it would seem logical to take advantage of the way our culture has been programmed to control other traffic.

\section{The Options Menu}

The Options menu of any application is well suited for commands and/or actions that are infrequently used or whose implications are significant enough that a little extra effort should be needed to activate them. For example, one would not want to inadvertently exit the software. Figure 4 shows an Options menu with items listed in order of most frequent anticipated use. In the author's experience, Volume is adjusted quite frequently, so it has been left off of the Options menu in favor of having its own button on the main display.

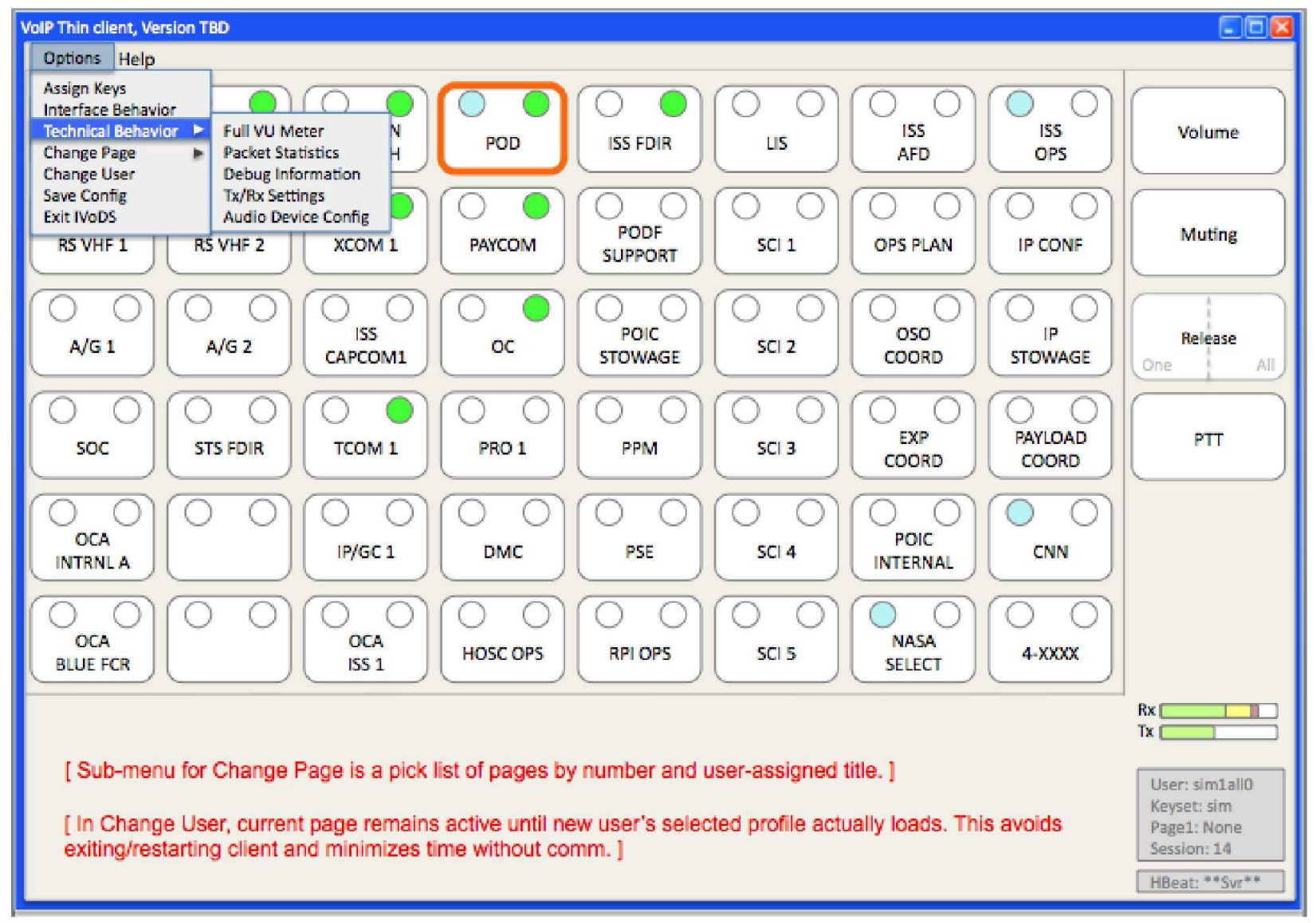

Figure 4 - "Options" Menu of the Suggested Interface 
The Demo System's Options menus had 'previous page' and 'next page' menu items without any dialogs to keep the user from inadvertently remapping their key assignments en masse. For most users (as opposed to voice system technicians), changing pages should be a rare occurrence. Use of a sub-menu (as opposed to having 'previous page' and 'next page' menu items) will help the user be deliberate. Allowing the user to provide page names would also make it easier for them to distinguish pages.

If one prefers to have a button on the main layout for changing pages, the author recommends designing it similarly to the "Release" button for interface consistency. In either case, after a new page is selected but before loading it, recommend having a confirmation dialog (X's indicate default settings):

Profile update for the page you are leaving:

$\begin{array}{lcc} & \text { Save } & \text { Erase } \\ \text { Talk/Monitor selections } & \square & \mathrm{X} \\ \text { Relative Volume settings } & \mathrm{X} & \square\end{array}$

\section{CHANGE PAGE CANCEL CHANGE}

\section{E. The Mute Function}

Users typically monitor several voice loops. If there is active traffic concurrently on all monitored loops, a user can quickly become overwhelmed. It's vital to be able to eliminate all but the most critical signal very quickly, hence the Mute function shown in Figure 5.

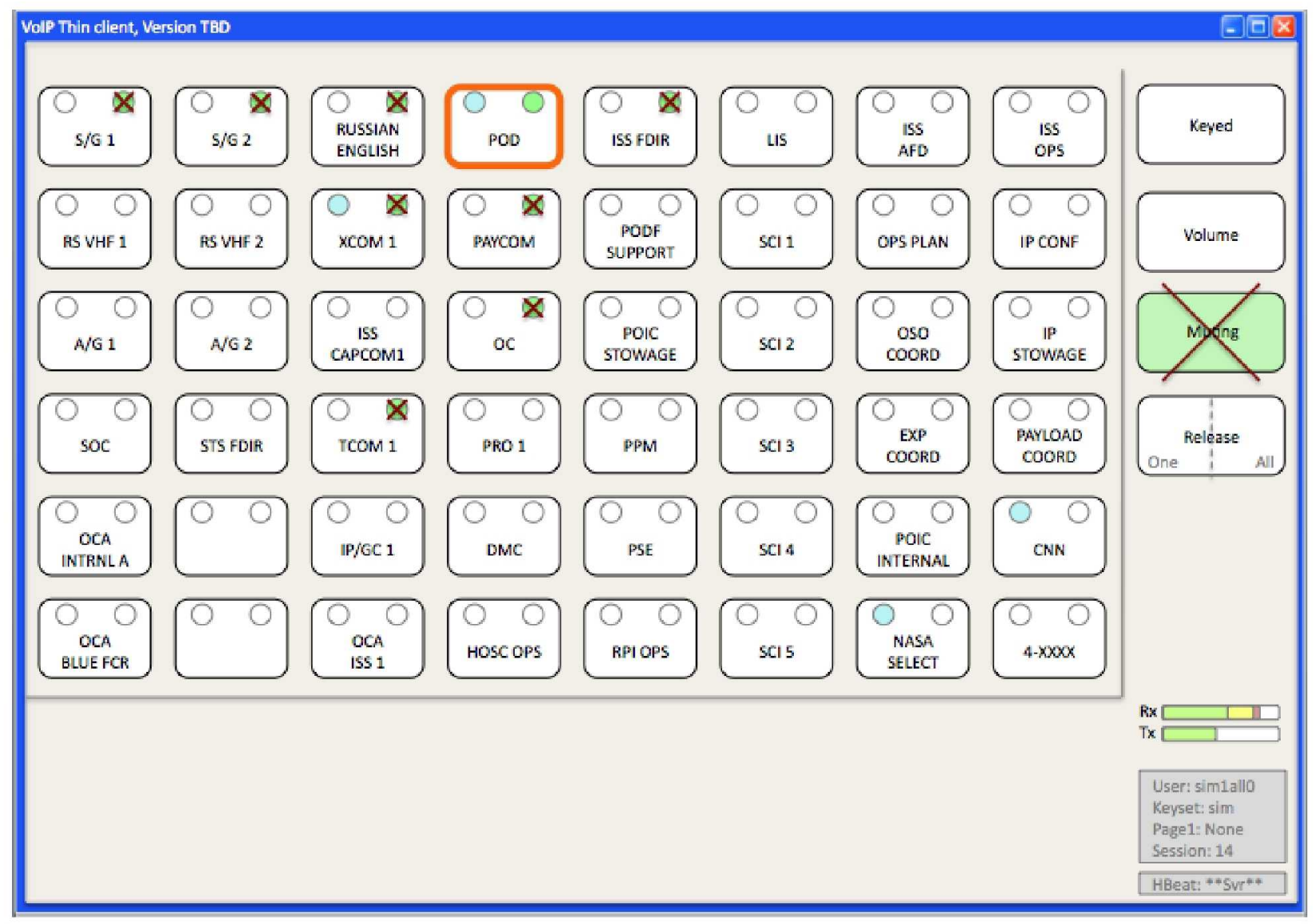

Figure 5 - "Mute" Function of the Suggested Interface 
When Muting is selected:

- All monitored loops except the one selected for Talk are muted, and ' $X$ ' appears over the Monitor indicators of monitored loops. It might be good to have the X's on the loop keys pulse or blink to enhance awareness of Mute state. This attribute could be established system-wide or one could allow user preference. Pulsing is a smooth increase and decrease of visual stimulus, in this case an ' $\mathrm{X}$ ', whereas blinking is a discrete on-off stimulus. The author recommends not having the symbolic ' $\mathrm{X}$ ' over the Muting key pulse or blink.

- Selecting a key unmutes it (' $\mathrm{X}$ ' disappears) but does not change the status of any other loop into Mute.

- The Mute state is added to the cycle of whatever Key-Press Behavior the user selected (see Figure 9 and associated discussion).

- Pressing the Muting key releases all muting.

\section{F. Demo System Volume Configuration and Loop Assignments}

Figure 6 illustrates the Demo System's provisions for the user to configure volume settings and to determine which voice loops will be associated with which keys in the visual matrix.
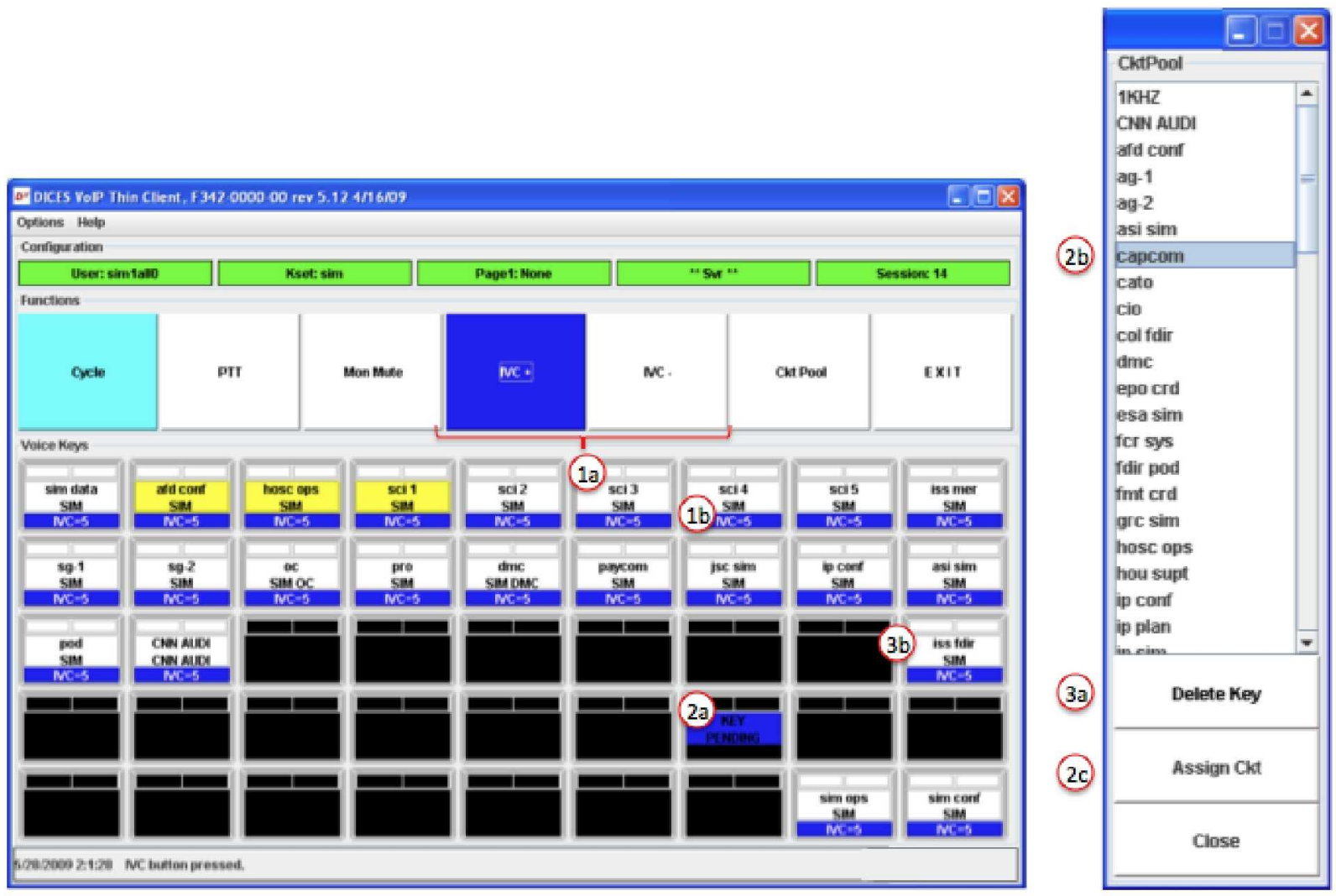

Figure 6 - Observations about Volume Control and Key Assignments of the Demo System

The numbered paragraphs below correspond to the numbered circles in Figure 6, and indicate areas the author selected for possible improvements.

1a. and $1 \mathrm{~b}$. When "Raise individual volume" or "Lower individual volume" (IVC+ or IVC-) is selected, clicking on a key in the loop matrix raises or lowers volume. The only cue that something is happening is the change in the number after "IVC $=$ " on the key. It is not clear which key is being changed until after changes are made, and it isfairly easy to change a value unintentionally and/or in an undesired direction. (Note - The author prefers the term "relative volume" to "individual volume", to drive home the distinction with respect to global volume, e.g., of a headset or speaker.) 
2a., 2b., and 2c. To assign a loop to a key, user typically selects the key and the loop, then clicks "Assign Ckt". If no key has been designated, the loop is assigned to the first open key (left to right, then top to bottom, beginning in the upper left corner). This can result in inadvertent assignments.

3a. and 3b. To delete a loop assignment, user has to click "Delete Key", then click on the key to de-assign.

4. The result of 2. and 3. is that loop assignment and deassignment processes are inconsistent internally and with respect to each other.

5. It would be helpful to be able to adjust global and relative volumes quickly without having to bring up different displays.

\section{G. Volume-Setting Scheme}

In considering Volume issues, the author sought a way to address the gamut of options in a comprehensive yet simple and unambiguous manner. The result appears in Figure 7.

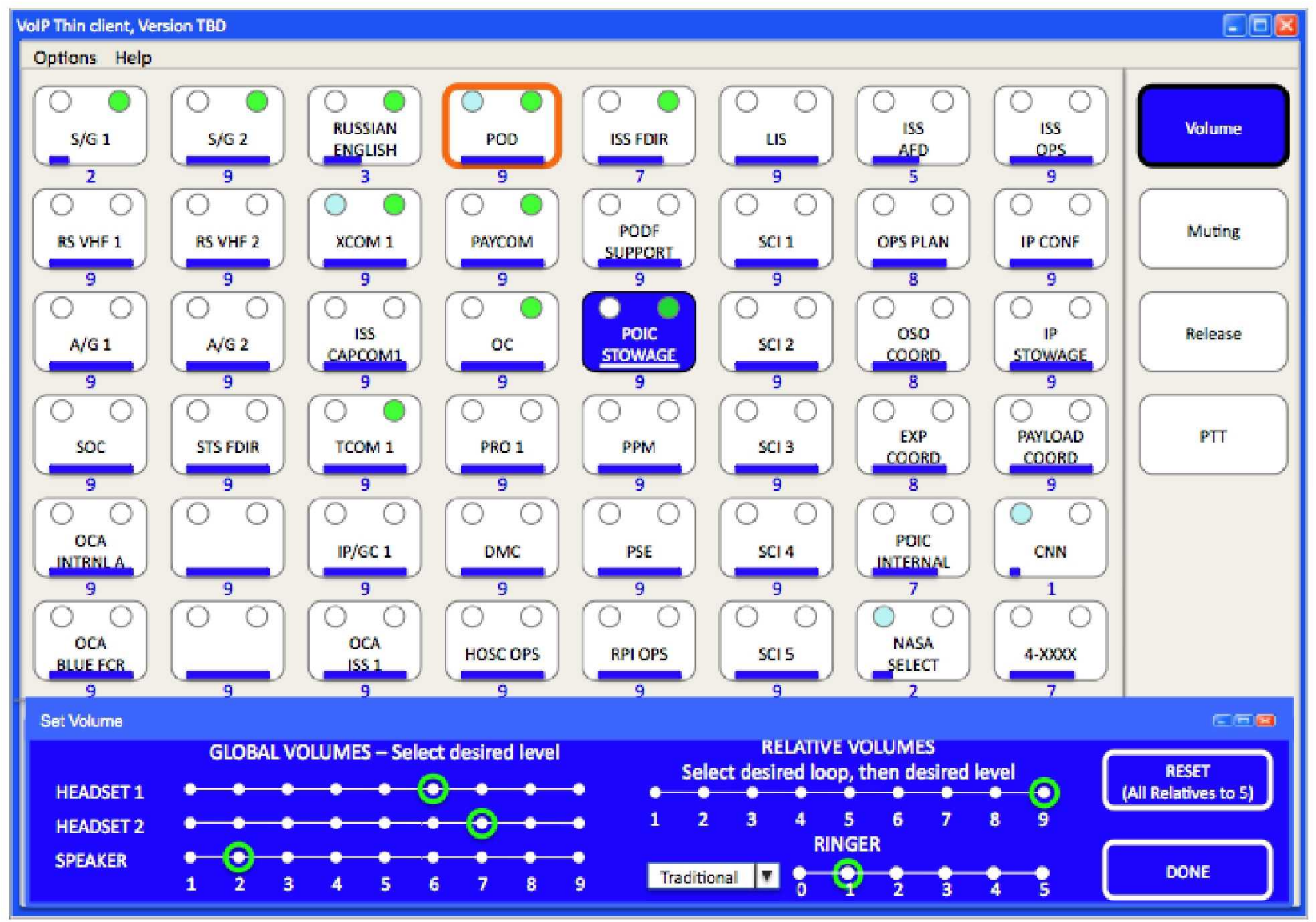

Figure 7 - Volume-Setting Scheme of the Suggested Interface

Keyset remains operational while configuring Volume. Use of a vibrant color to signify a configuration mode encourages the user to not stay in that mode for too long. All vibrant color disappears when DONE is selected and client returns to normal operation.

Volume levels may be set by clicking on desired level or by dragging the focus circle.

To the greatest extent possible, the keys perform as though the user was in normal operational mode. 
- Talk, Monitor, and Listen status change per user-specified Key-Press Behavior selection.

- Muting and Release keys function as usual.

- The most recently pressed matrix key, if in Talk or Monitor (and not Muted), is highlighted solid with reverse text, which tells the user that they can set relative volume for that key.

Popup dialogs appear for the following selections:

DONE button or Close Window or Volume Key

UPDATE current profile USE settings without saving

CREATE new profile REVERT (ignore these adjustments)

CANCEL (keep adjusting)

RESET - Do you really want to set all relative volumes to 5? YES CANCEL

[If Yes] Are you absolutely sure you want to set all relative volumes to 5? YES CANCEL

PAGE - Go through DONE dialogs, proceed to Previous/Next Page unless Cancel

Some system-level issues remain: Does the telephone ringer sound in headsets, on the speaker, or both? Is this a system-wide setting or an individual profile option? The example shown lets the user select a ring sound.

\section{H. Assigning Voice Loops to Matrix Keys}

Figure 8 shows how a user might assign voice loops to suit their personal preference, subject to privileges assigned by the system administrator.

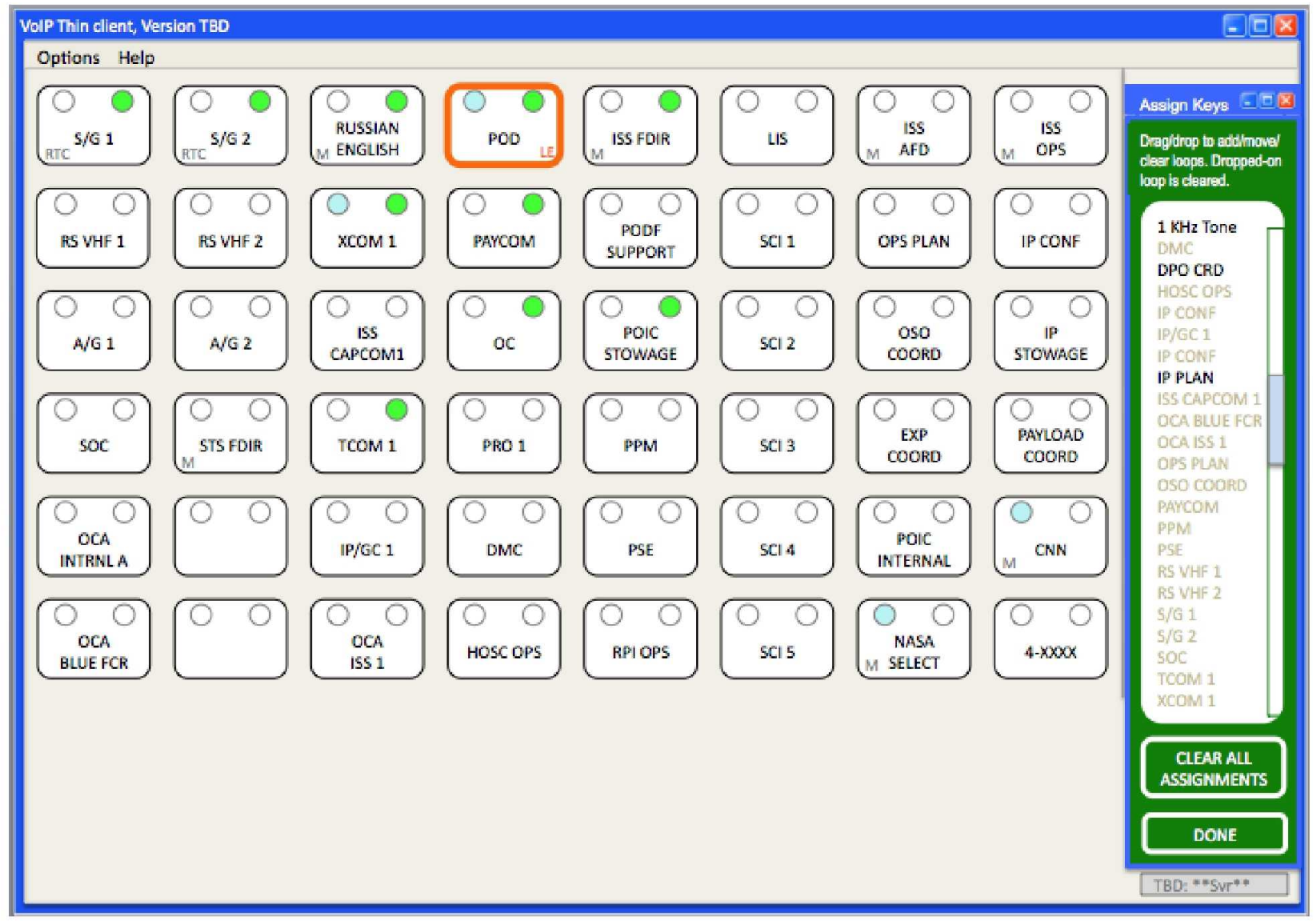

Figure 8 - Assigning Voice Loops to Matrix Keys of the Suggested Interface

11

American Institute of Aeronautics and Astronautics 
The keyset remains operational while assigning loops. The "Assign Keys" window can be moved for access to command keys. Large block of color encourages user to not stay in this mode for too long.

Selecting-and-holding a loop matrix key signals intent to change the loop assignment for that key, while momentary select activates the Key-Press Behavior per user selection.

In the "Assign Keys" window:

- Loops already assigned on current page show in a light color.

- Loops that the user is not authorized to assign should either be omitted or also shown in a light color.

- While not depicted here, there could be indications as to user authorization for Talk capability.

If audio summing problems can be avoided, one could allow assignment of one loop to multiple keys. This is not an envisioned need for MSFC, but in some settings, the capability could be useful for user convenience and visual ease.

The Phone key is always in the same location on the matrix (lower right corner in this example).

Popup dialogs and options appear for the following selections:

DONE or Close Window

UPDATE current profile USE settings without saving

CREATE new profile REVERT (ignore these adjustments)

CANCEL (keep adjusting)

CLEAR ALL ASSIGNMENTS - Do you really want to clear all key assignments? YES CANCEL

[If Yes] Are you absolutely sure you want to clear all assignments? YES CANCEL 


\section{User-Configured Interface Behavior}

Some aspects of interface behavior should be standardized so that users have much experience in common, to make consistent technical support possible, and to simplify training requirements. However, users are often more effective if they are allowed some level of custom fit. Figure 9 attempts to strike a reasonable balance.

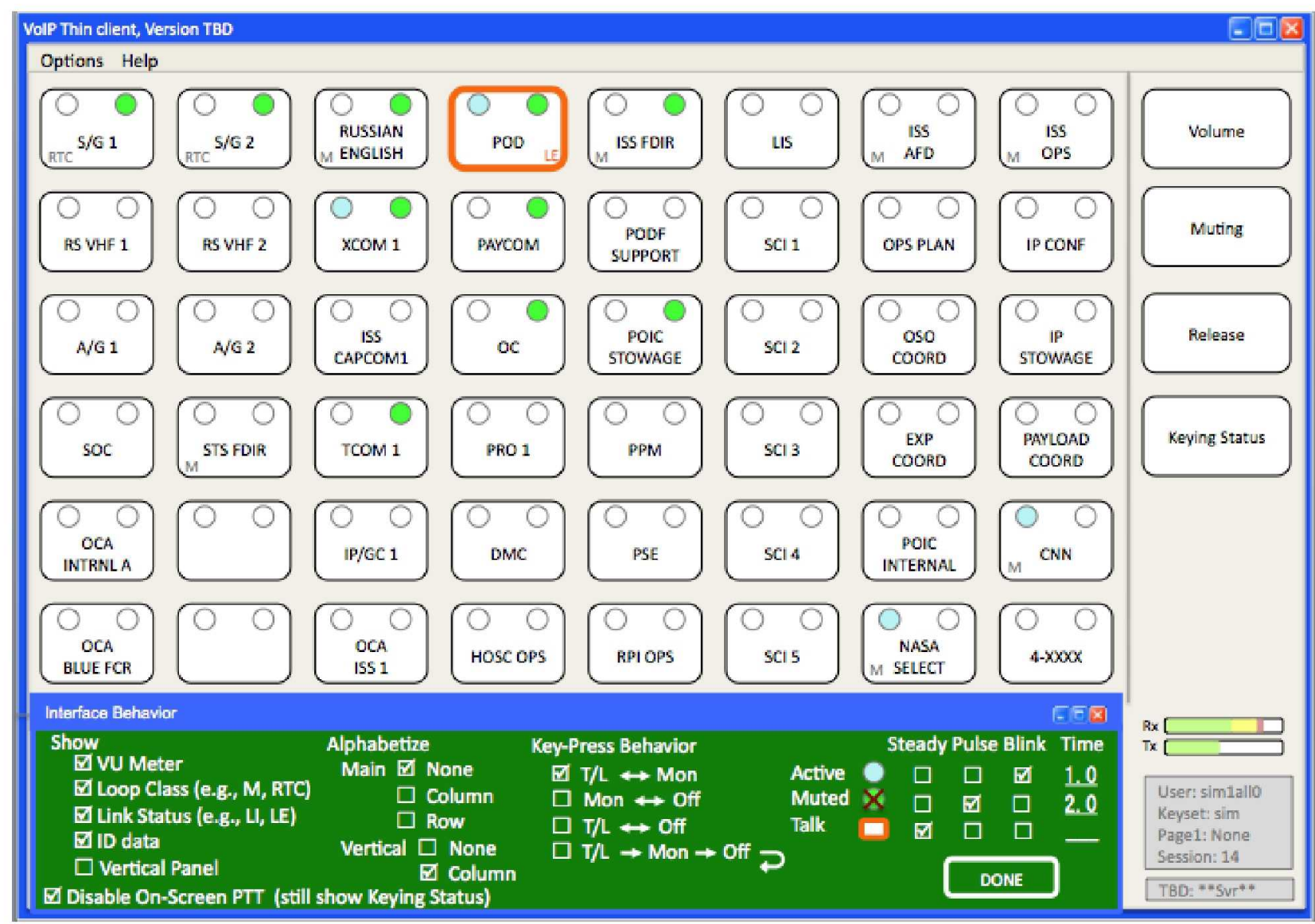

\section{Figure 9 - User Settings for Behavior of the Suggested Interface}

The keyset remains operational while configuring appearance. Large block of color encourages user to not stay in this mode for too long.

"Show" items and "Disable On-Screen PTT" are click-on, click-off.

All others are select-only - clicking one box deselects other option(s) for that function.

Symbolic colors should be set group-wide; colors should be identical across operating systems. This may require a translation table.

Graphics just to the right of the Active/Muted/Talk titles in the Interface Behavior window exhibit their specified behavior, e.g., steady, pulse, or blink (in case there is no instance on the matrix at the time).

There should always be a large indicator, triggered by feedback from the voice switch, that lights up whenever the client is keyed. This will remind the operator that their voice is going out and, if it fails to light up, signifies transmission failure. Many operators may not want the risk of having an on-screen PTT, hence the "Disable" option in the lower left corner. If Disabled, PTT button wording changes to "Keying Status", but light-up behavior is the same in both settings. 
Note that displaying Vu Meter, Loop Class, Link Status, and ID data are optional. However, Heartbeat is always displayed in the very lower right corner of the keyset, because this will be the quickest sanity check of keyset health for both the user and technician support.

Popup dialogs and options appear for the following selections:

DONE button or Close Window

UPDATE current profile USE settings without saving

CREATE new profile REVERT (ignore these adjustments)

CANCEL (keep adjusting)

CLEAR ALL ASSIGNMENTS - Do you really want to clear all key assignments? YES CANCEL

[If Yes] Are you absolutely sure you want to clear all assignments? YES CANCEL

\section{J. Telephone Interface}

Integration of telephone capability, typically via a Private Automated Branch Exchange (PABX), into a voice switch keyset can be both a simplification and a complication (or, if one prefers, both a blessing and a curse). Figure 10 proposes an interface intended to minimize confusion.

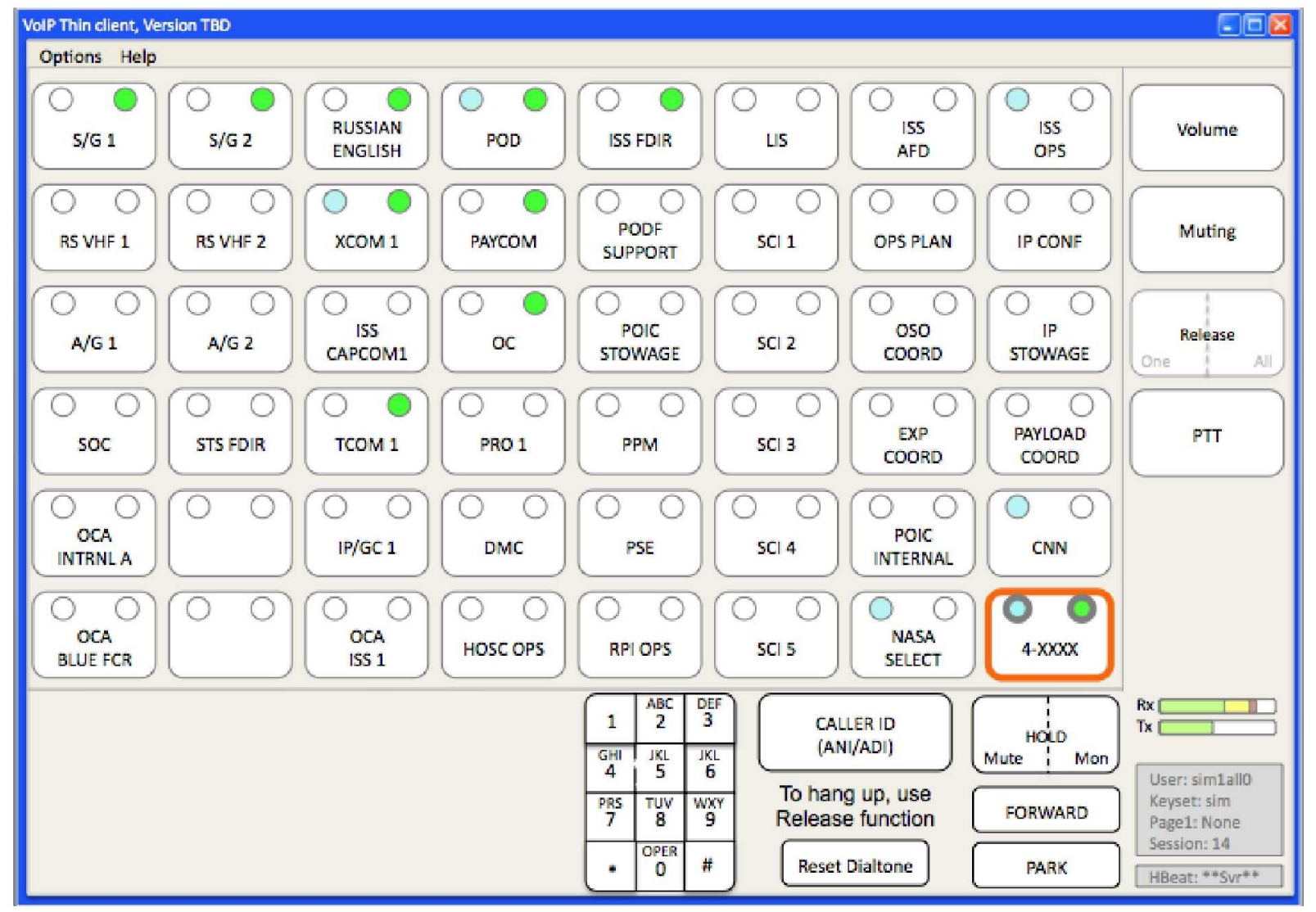

Figure 10 - Telephone Operation of the Suggested Interface

Incoming Call (prior to answering)

- Caller ID window appears with caller ID info (ANI/ADI = Automatic Number ID/Automatic Address ID)

- Telephone key border thickens and flashes using Active indicator color

- Ringer rings per user and system configuration

14

American Institute of Aeronautics and Astronautics 
Outgoing Call

- In this concept, behavior is like a conventional phone

- Pressing the Phone key is like taking phone off hook - User hears dial tone

- Dialed digits appear in Caller ID field

Anytime user activates the Phone (answer incoming call or place outgoing call)

- Phone key border behaves as for any other Talk-selected loop

- Phone keypad, Caller ID field, command buttons, and hangup reminder appear, and remain on screen until call ends (hung up, forwarded, or parked)

- To prevent inadvertent call hangup, pressing the Phone always toggles between Talk/Listen and Monitor states, i.e., the console operator must use the Release function to hang up.

\section{Entering HOLD}

- Pressing HOLD (Mute) suppresses Phone audio from headset/speaker. Pressing HOLD (Mon) allows user to hear Phone audio, User can toggle between Mute and Mon Hold by pressing the appropriate side of the HOLD key.

- With Phone in Talk mode, a key press on the matrix that puts another loop into Talk mode automatically puts Phone into Hold with Monitor. (Could change default to Hold with Mute, or make option part of user profile)

Interface Behavior in HOLD

- Appropriate side of HOLD key lights up with color/behavior specified in Interface Behavior.

- Loop monitored indicator on Phone key adopts Monitor or Mute color/behavior as for any other key.

- Keypad, Caller ID, and Phone command button outlines go to gray until Phone re-enters Talk mode.

- User must press Phone key to get circuit in Talk mode in order to transmit on Phone, forward call, or park call.

A call can be hung up at any time by using the Release function. Note that is a two-step process, which should ensure that the user meant to end the call.

If a call is forwarded or parked, an advisory dialog appears to the left of the keypad showing forward/park destination. Dialog goes away only when user presses an OK button within it, even if other Phone fields have gone away. (This preserves information to give user time to jot it down or to use the information to re-establish the call.)

3-Way/Multipoint Calling and Call Waiting are not shown in this concept. The capability could be useful, but the author's experience suggests that, keyset operations being reasonably complex as they are, such features might best be left to a separate, standard telephone ("black phone" in NASA mission control parlance) that is independent of the mission voice switch. (There are times when the act of dealing with a physical handset makes it easier to keep things straight.)

\section{K. User-Controlled Phone Patch}

Patching of outside phone calls into voice loops is typically done by system operators or technicians. However, a simple "Create Phone Patch" function for non-technicians could be valuable in a number of settings, as long as operational lines of authority and permission for the patch are clear. (The author's experience has often been that occasions calling for a phone patch often occur at a time when many other things are happening and technicians are unavailable.) An interface for simple phone patches is shown in Figure 11. 


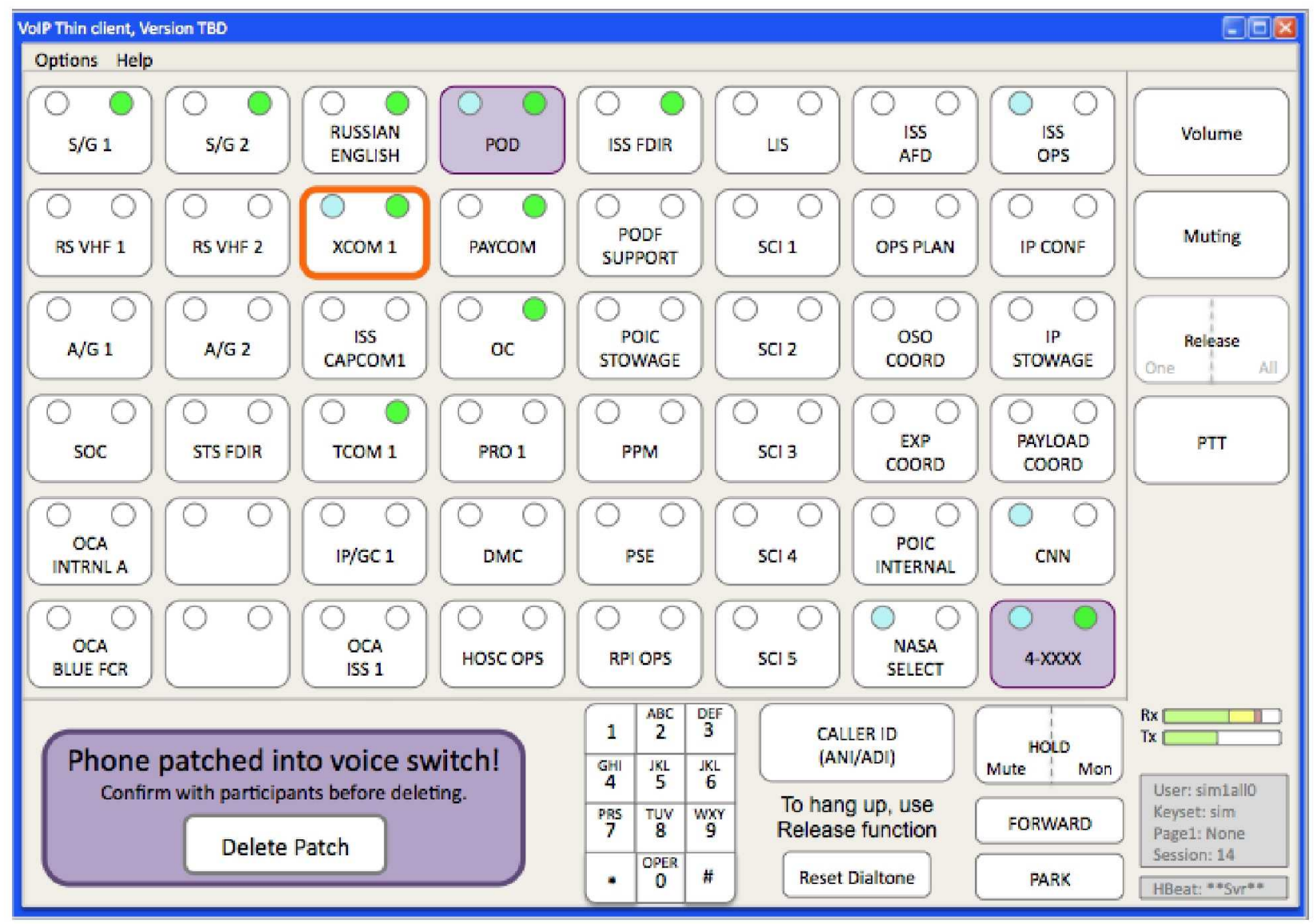

Figure 11 - User-Controlled Phone Patch via the Suggested Interface

Patch capability could be implemented as an item on the Options menu or via a "Create Phone Patch" button that appears when the Phone is activated by pressing the key in the lower right corner of the loop matrix. In either case, the function should be invisible unless the user has patch privilege.

Rules of Engagement:

- Simple privilege - User can patch into any of their Talk-capable loops

- Complex privilege - User can only patch into Talk-capable loops specifically called out in their profile

- Any loop patched into must be visible on user's current page

- To initiate patch, Phone must be in Talk mode with a party on the line.

A Phone Patch sequence could be:

- Initiate 'Create Phone Patch' command.

Dialog - 'Select loop to patch into'

Depending on implementation, user clicks on desired loop or selects from pick list

- On loop selection, Dialog -

Are you sure you want to patch your Console Phone into [Loop Name]? YES RE-SELECT CANCEL

If Yes, 'Are you REALLY sure you want to complete this patch?' YES RE-SELECT CANCEL

If RE-SELECT, go back to 'Select loop to patch into'

If CANCEL, exit patch mode completely

- While the patch is active:

o The Phone circuit and patched loop are shaded as shown in Figure 11. (Purple is for Patch!) In this example, the user creating the Patch has gone to Talk on a different loop.

o The HOLD function works as usual with respect to the keyset user, but the Phone call remains live on the patched loop. 
o The "Phone patched into voice switch!" warning dialog remains until the patch is deleted.

o If RELEASE function is applied to the Phone key the 'Delete Patch' logic below is activated

o If RELEASE function is applied to the patched loop, the loop is muted with respect to the keyset user but does not affect the patch.

o If CHANGE PAGE function is activated,

Dialog - "You must end phone patch before changing page OK"

o If party on other end of Phone call hangs up

Patch is automatically deleted, key shading goes away, Phone goes to inactive state.

Dialog - "Phone caller hung up or call dropped" - remains until closed by user

o "Delete Patch" button press activates Dialog -

Are you sure you want to delete your phone patch? YES NO

If YES, key shading goes away, client prompts user to keep or drop Phone call.

If NO, client reverts to state just prior to 'Delete Patch' command.

\section{Vertical Panel Options for the Demo System and the Suggested Interface}

The Demo System included a vertical panel scheme, which could be quite valuable when a workstation supports multiple operations functions and screen real estate is at a premium. This is typical of remote sites that often do not have funding for dedicated keysets with embedded processors. Figure 12 shows how the Demo System's vertical panel might be implemented using the suggested interface's motif and philosophy.
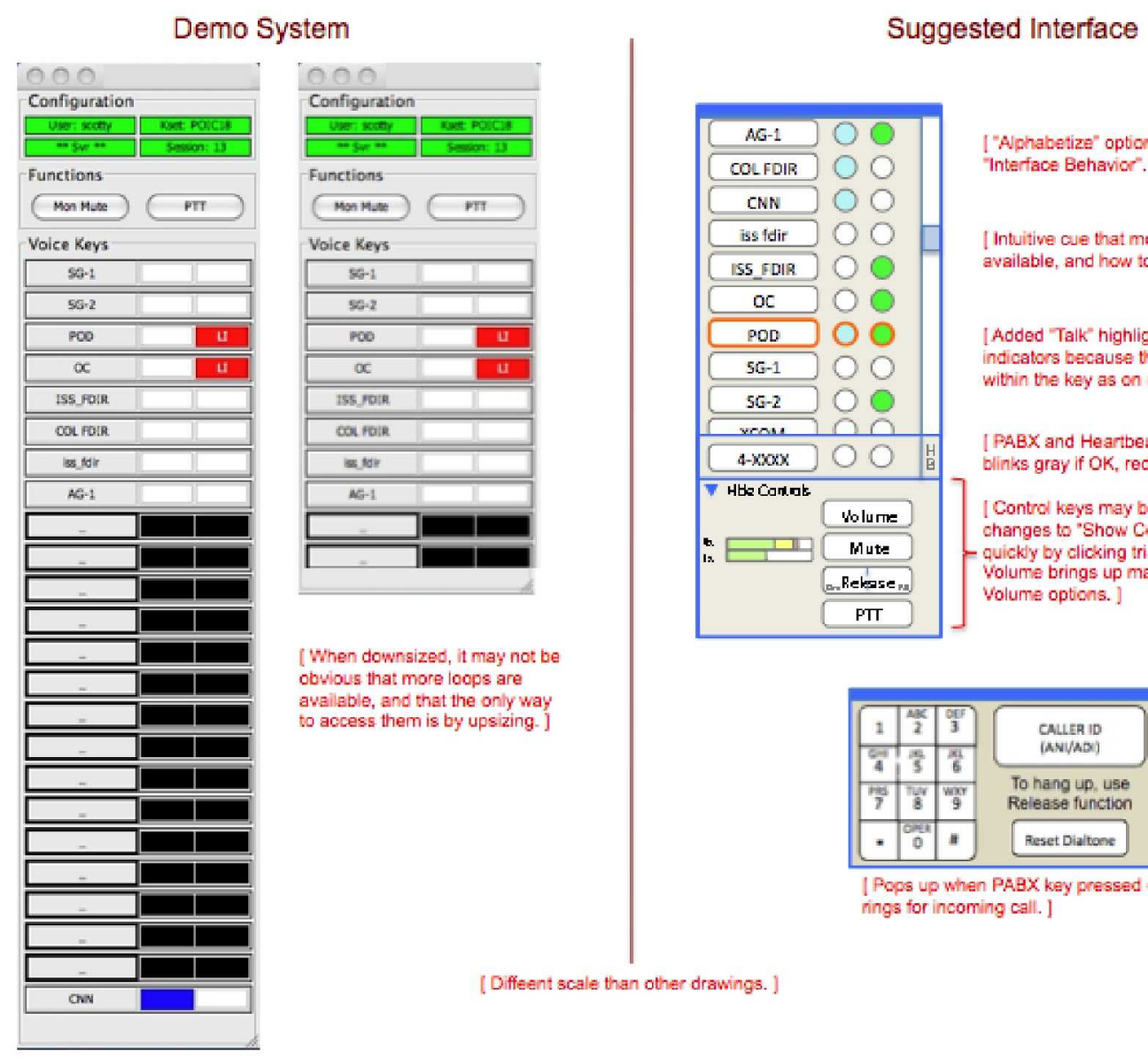

[ When downsized, it may not be obvious that more loops are awaliable, and that the only way to access them is by upsizing.

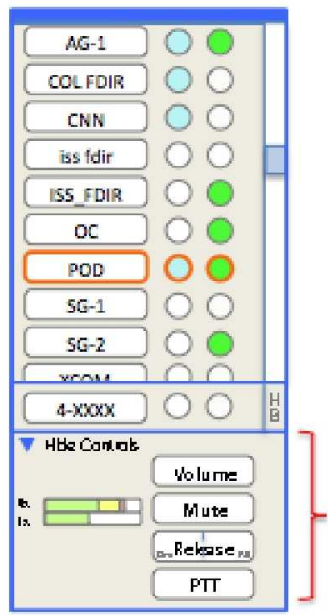

[ "Aphabetize" option selected in "Interface Behavior". ]

[ Intuitive cue that more loops ane available, and how to reach them. ]

[Added 'Talk' highlighting around indicaters bacause they're not contained within the key as on matrix-style display. ]

[PABX and Heartbeat always visible. HB blinks gray if OK, red if excection. ]

I Control keys may be hidden (text changes to "Show Controls"), but available quickly by clicking triangle. Selecting Volume brings up matrix display with Volume options. ]

Figure 12 - Vertical Panel Options for the Demo System and the Suggested Interface 
For the suggested interface, note that command keys:

- Are in the same positions relative to each other as on the matrix layout (for consistency).

- Are offset from the loop keys (to reinforce the functional difference).

An extension of this concept would be to provide an editor that lets the user build their own key layout (not just key assignments) so that keys are organized and shown in logical groupings based on the user's individual needs and operational patterns.

\section{Conclusion}

Using a dedicated touch-screen device or a computer application window as a mission voice system keyset provides tremendous flexibility in keyset design and permits significant modification of the interface and its behavior at relatively low expense, since hardware need not be replaced. Most mission voice switch users can benefit greatly by having a simplified interface that focuses on operational functions and minimizes display of technical voice switch details. (System operators and technicians whose job is to manage and troubleshoot the voice system itself obviously need more complicated displays.)

Keysets should remain as operational as possible during configuration activities, since calls from other operators may come at any time.

Because voice systems are an integral part of operations control and may be used incessantly by some personnel, often during periods of intense communication and mental pressure, there is merit in fine tuning the interface design. Sharp axes cut clean and make the loggers efficient.

\section{Acknowledgments}

The author thanks Jim Bathurst, Patty McMahon, and Joe Fittipaldi of the MSFC Mission Operations Laboratory's Payload Displays Review Team for their insight on human factors, and Steve Calvelage and Carl Ellis of the Huntsville Operations Support Center (HOSC) Systems Engineering team for their help in setting up demonstration hardware and software and for hours (upon hours) of discussions about voice switch details and possible interface behaviors.

\section{References}

Not applicable. 


\section{Suggestions for Layout and Functional Behavior of SOFtWARe-BASEd Voice SWitch Keysets}

Introduction

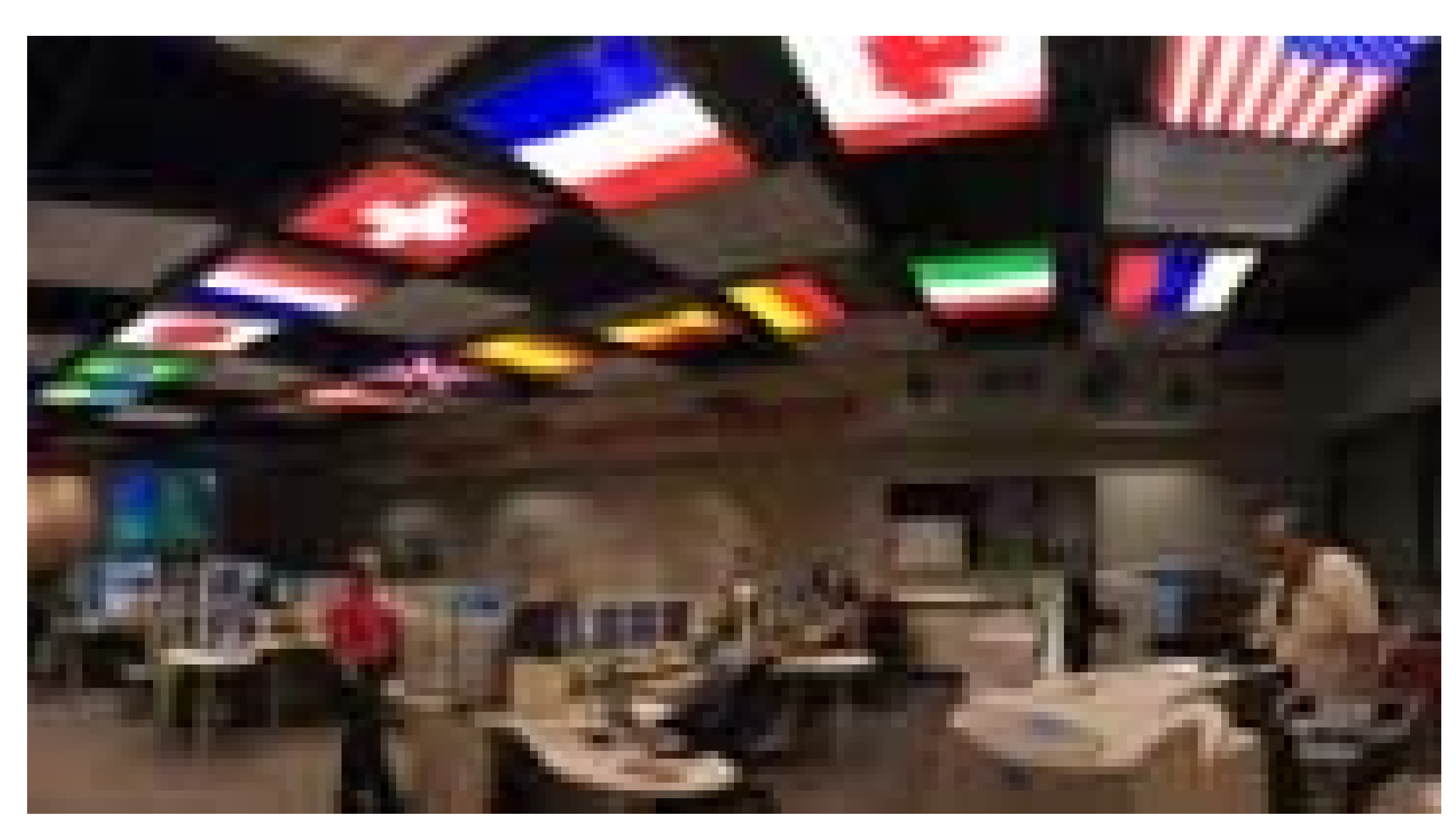

Basic Features of Suggested Interface (SI)

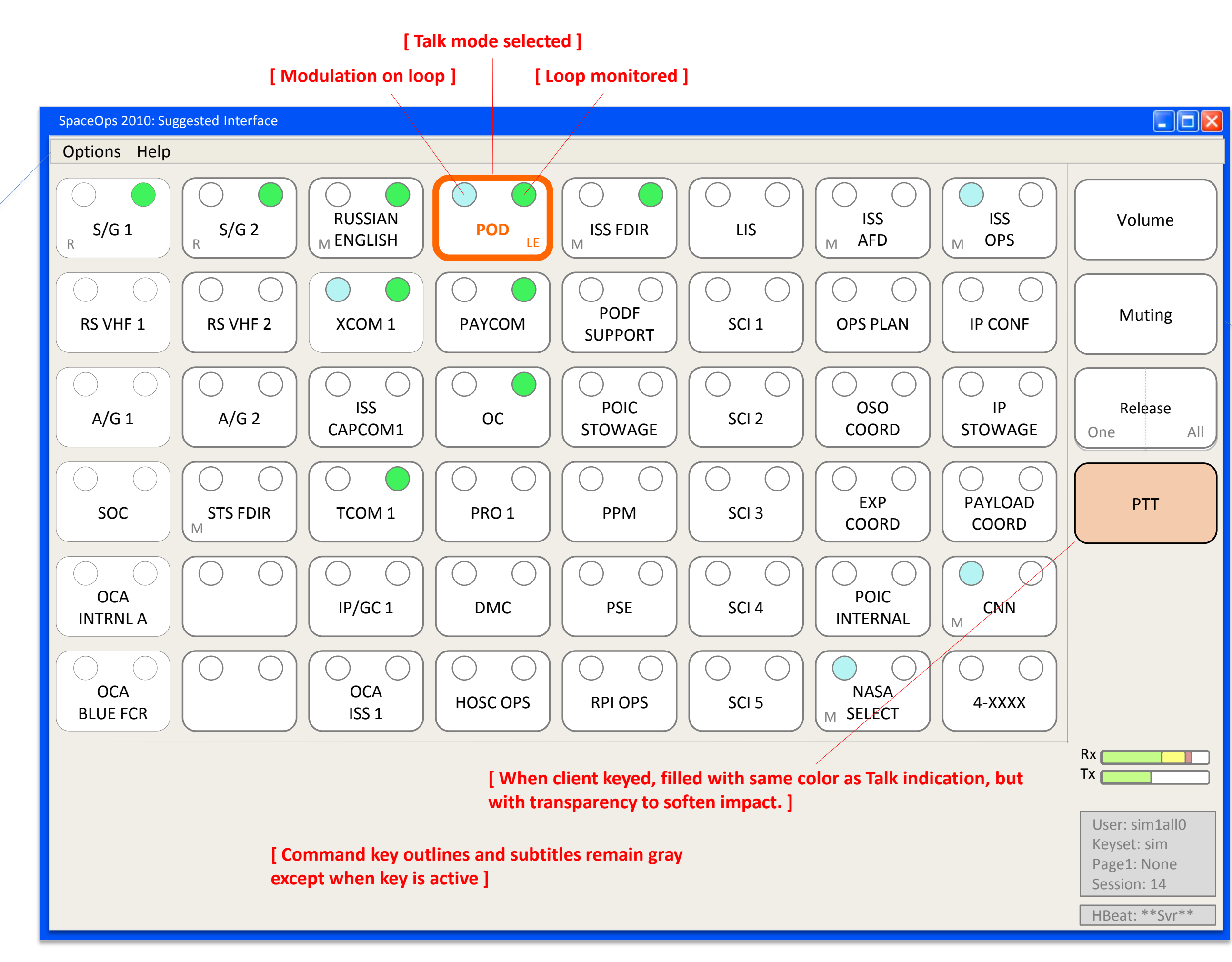

Mute Function

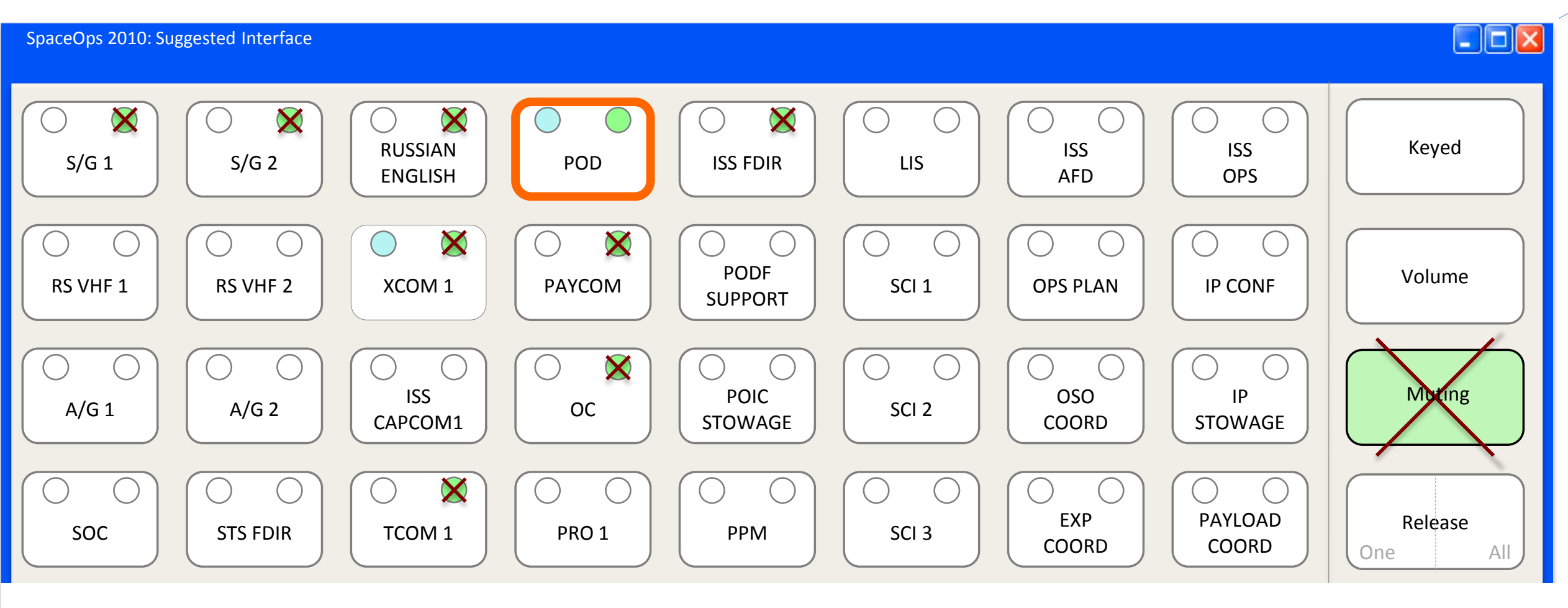

Options Menu

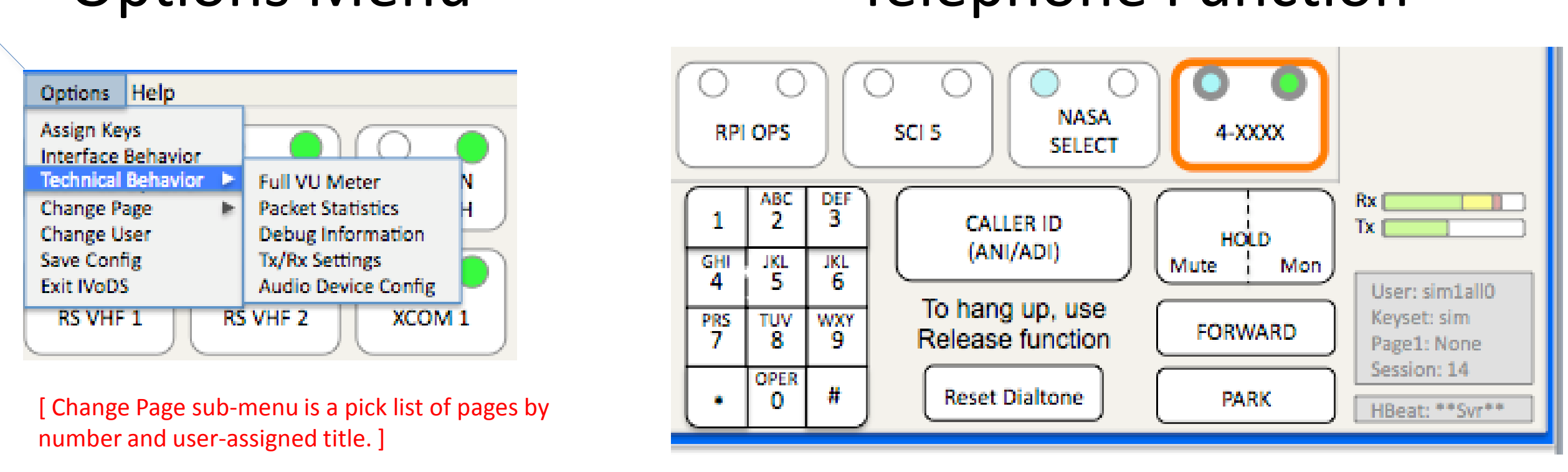

MSFC Legacy Keysets

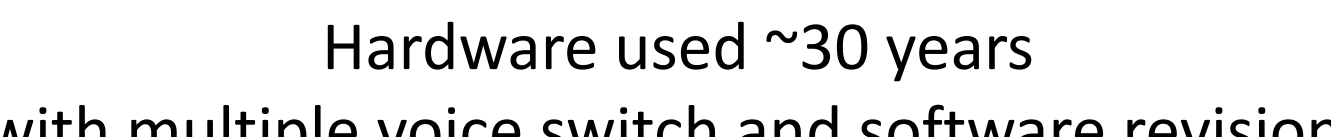

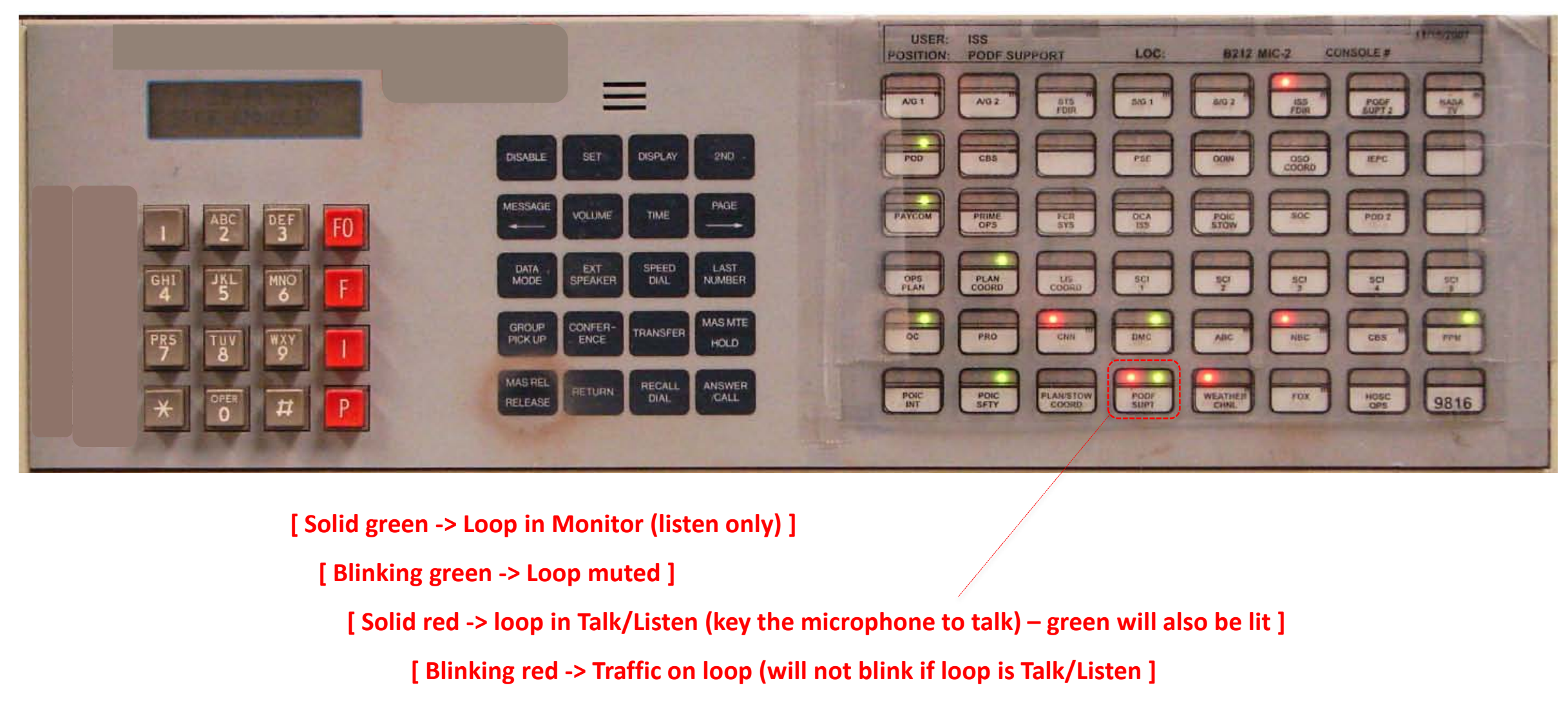

Demo System Volume Control \& Loop Assignment

Most users adjust volume fairly often, loop assignments rarey

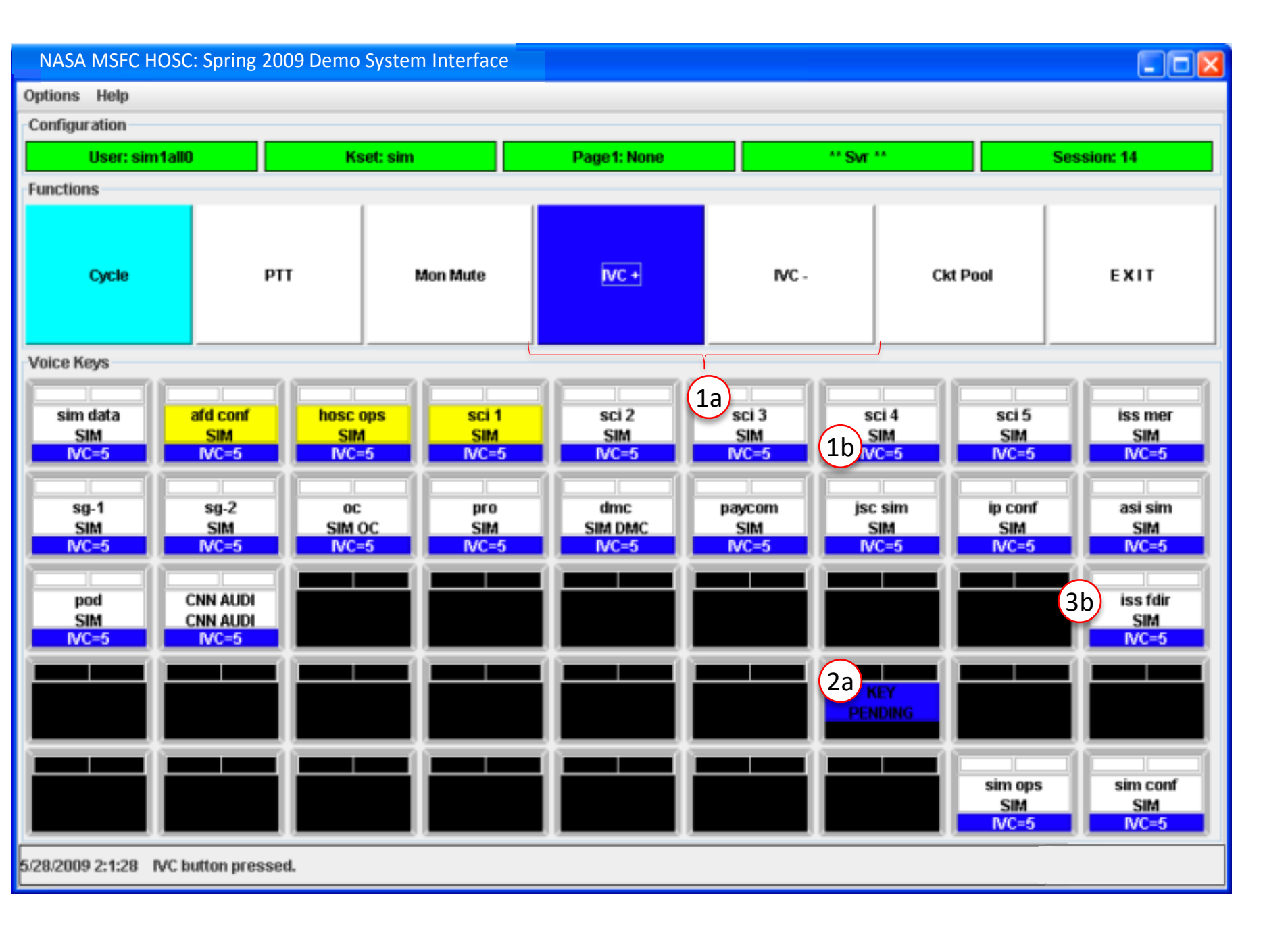

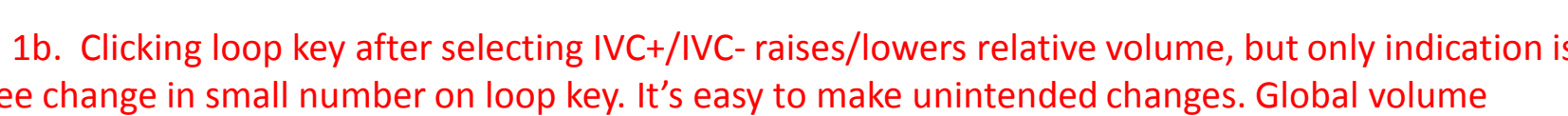

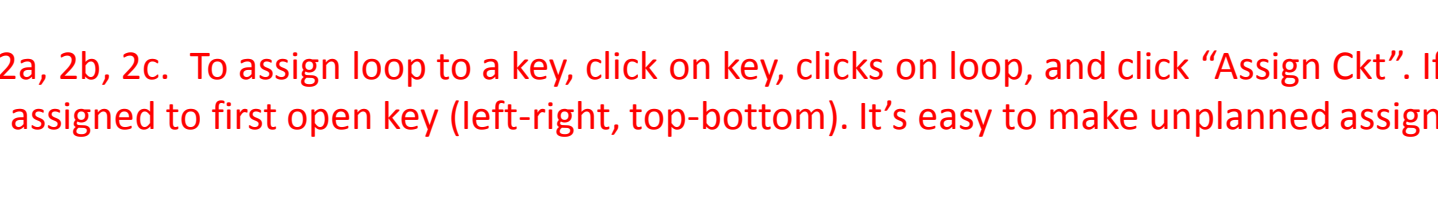
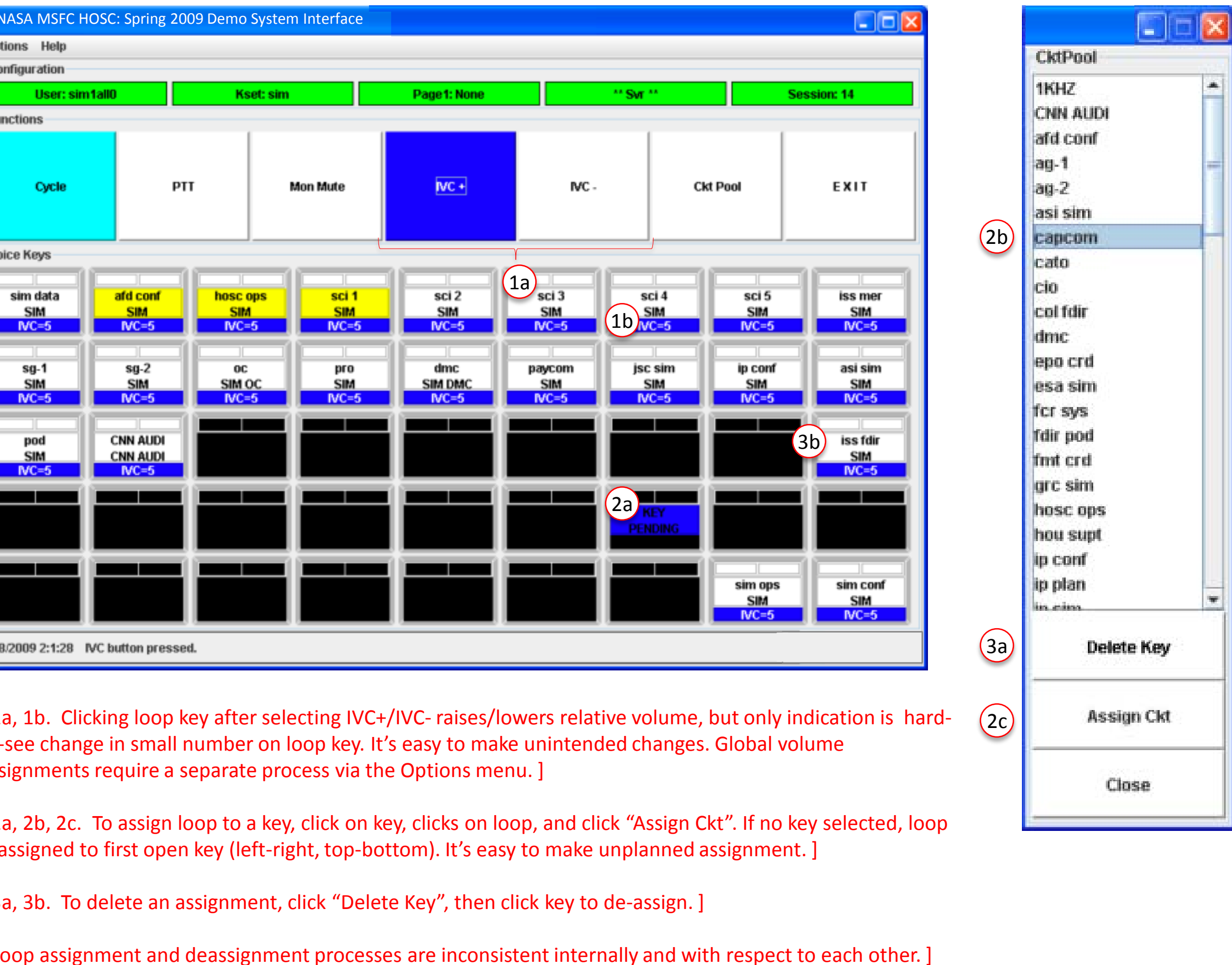

All volume settings can be viewed and adjusted concurrently.
During config, Talk \& Monitior selections frozen but PTT works.

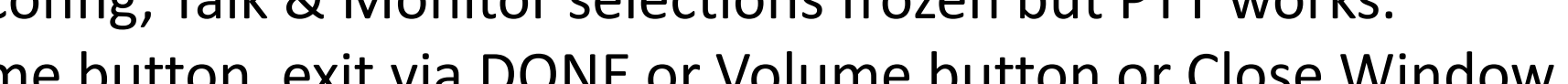

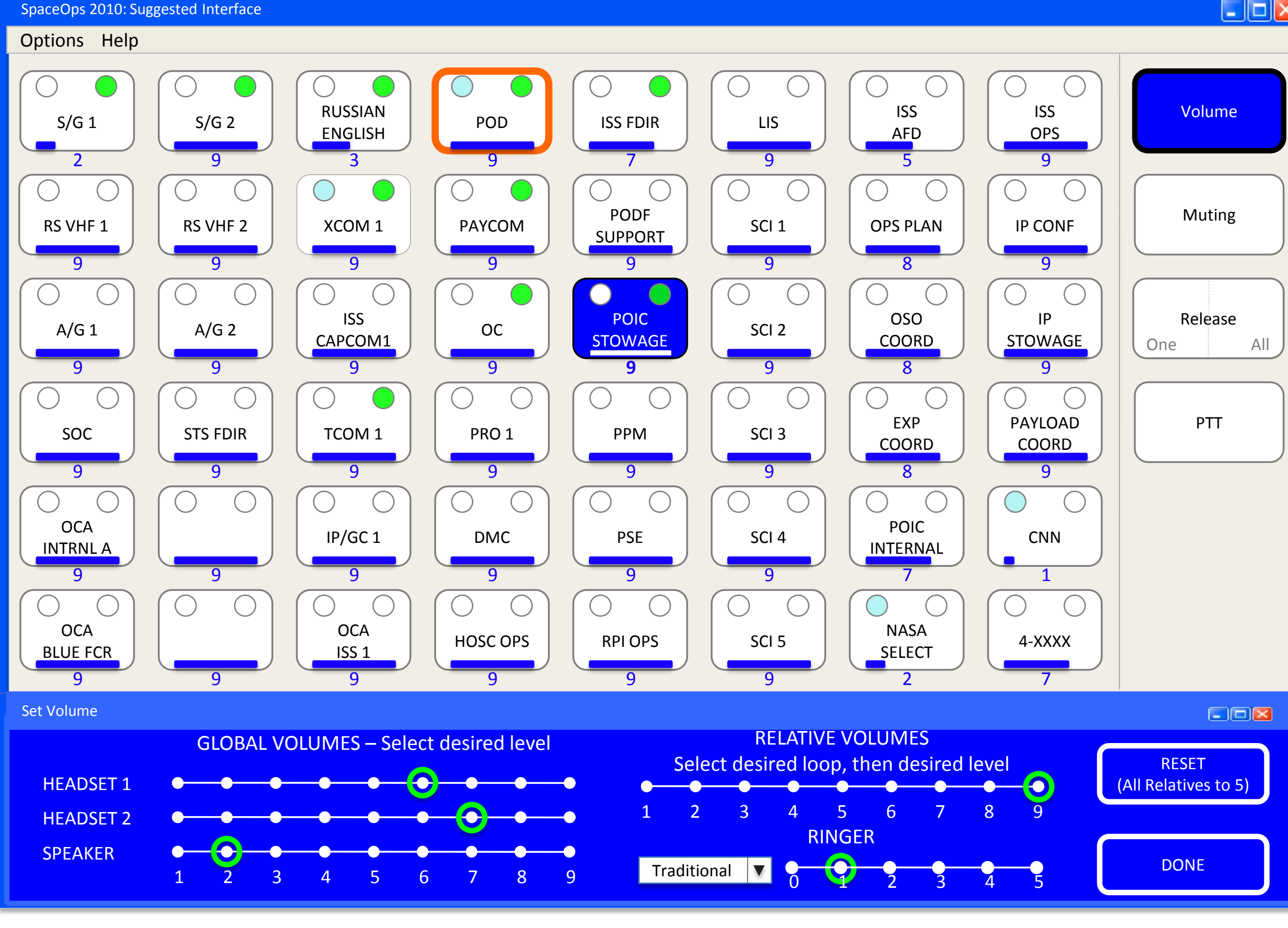

Vertical Panels
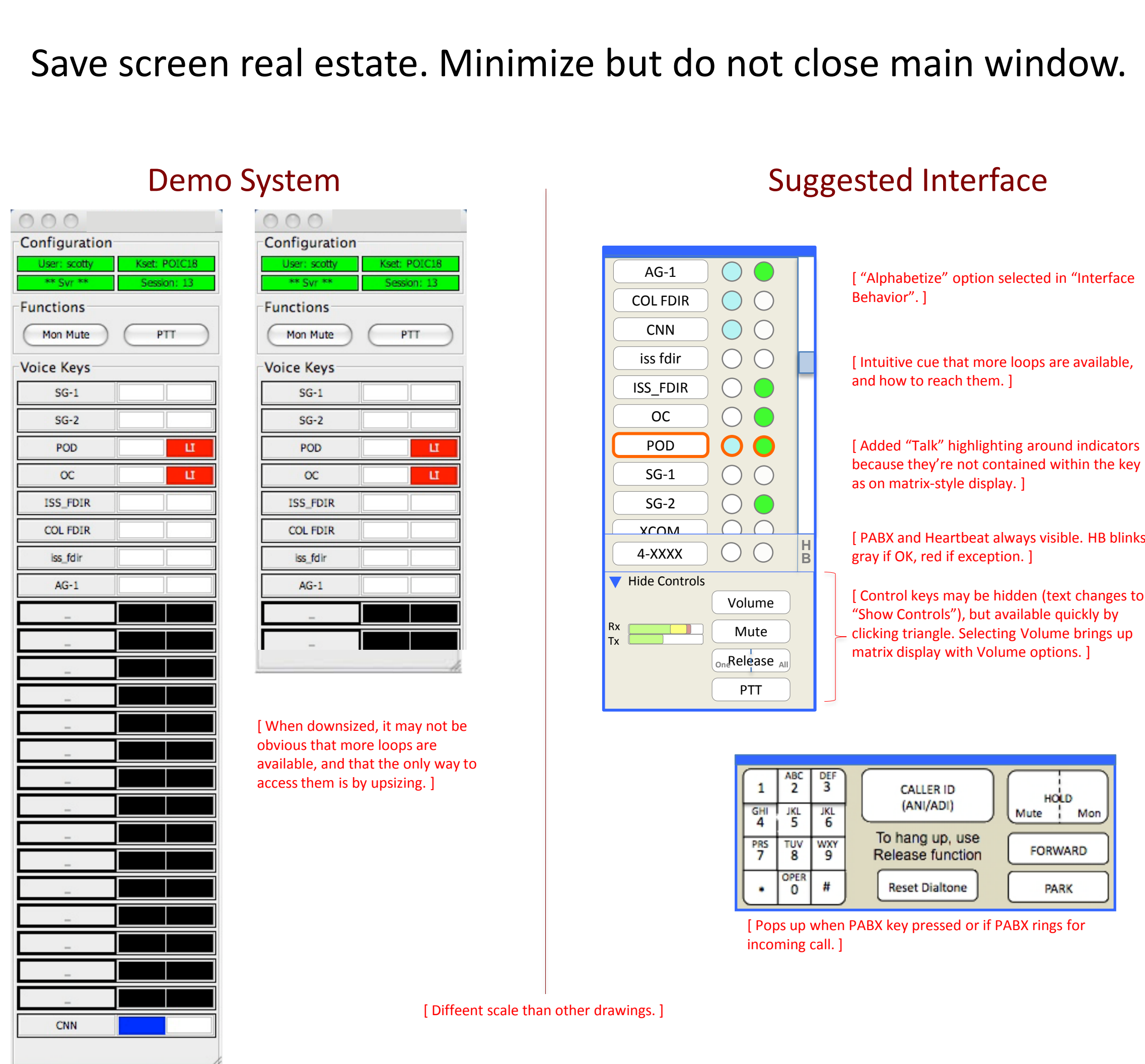

Spring 2009 Demo System Interface

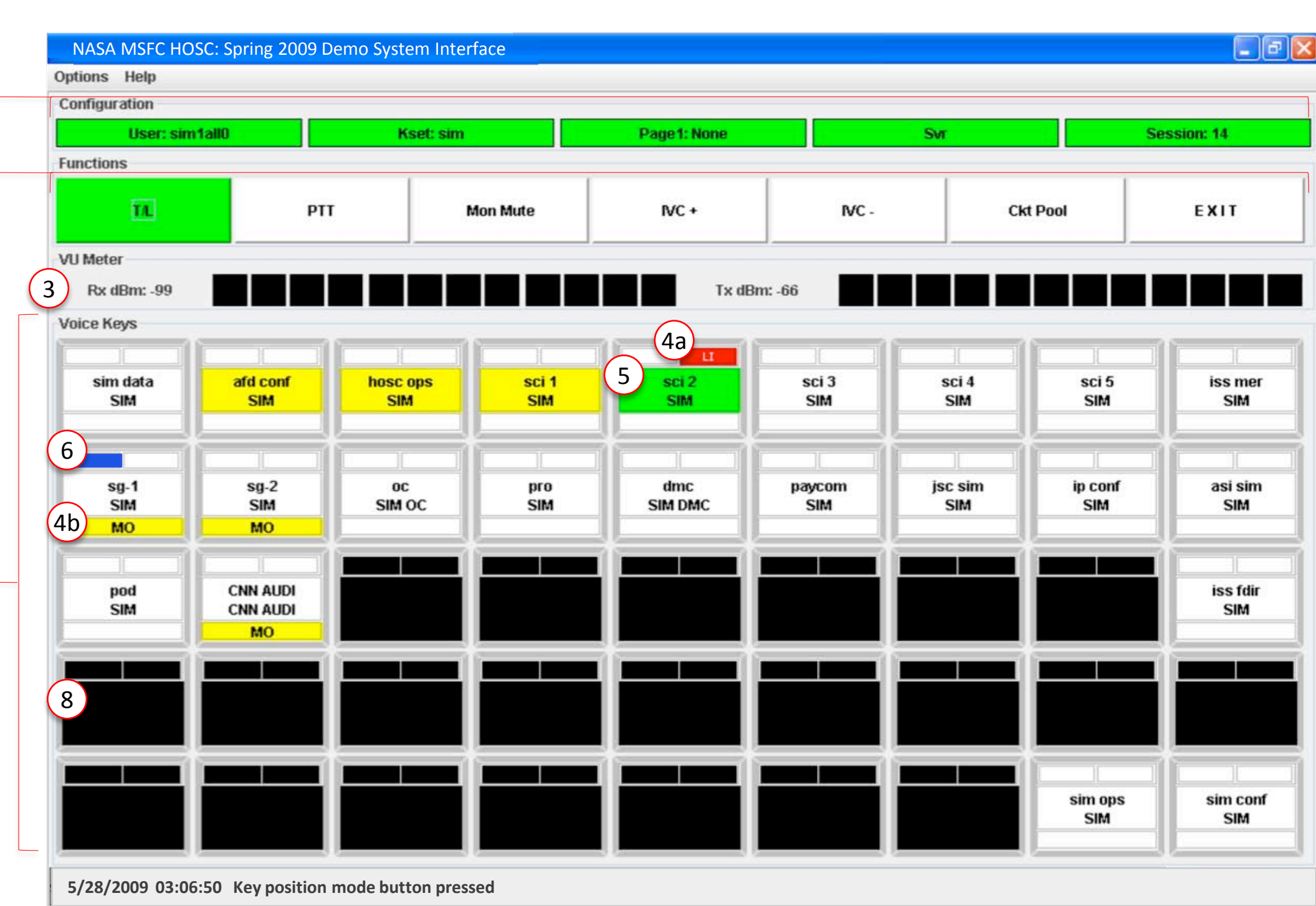

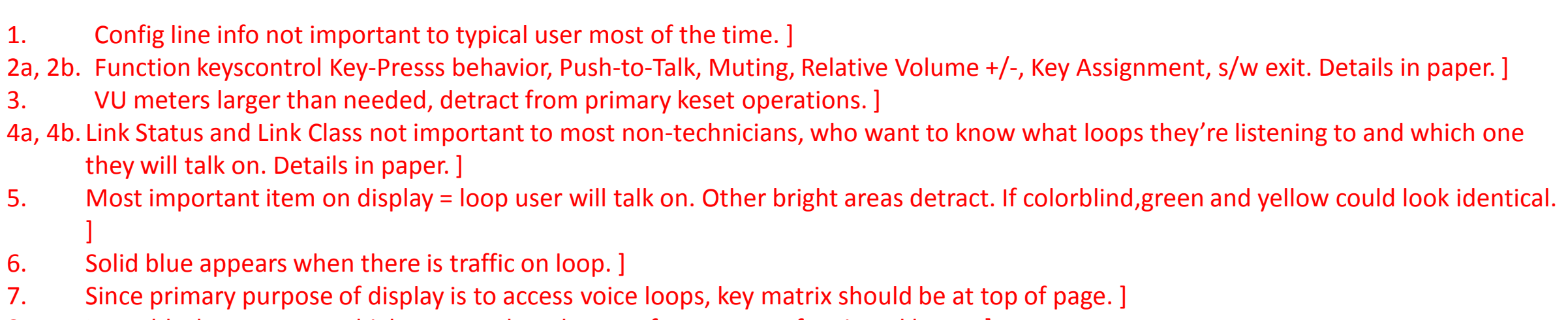

SI - Setting Volumes

SI - User-Controlled Phone Patch

hugened in MSFC's current environment, but tool could be invaluable during
intensive opsif lines of authority/germission are well-established.
SI - Assigning Keys, Customizing Behavior

Keyset fully operational while configuring.
Enter config modes via Options menu, exit via DONE button or close Window.

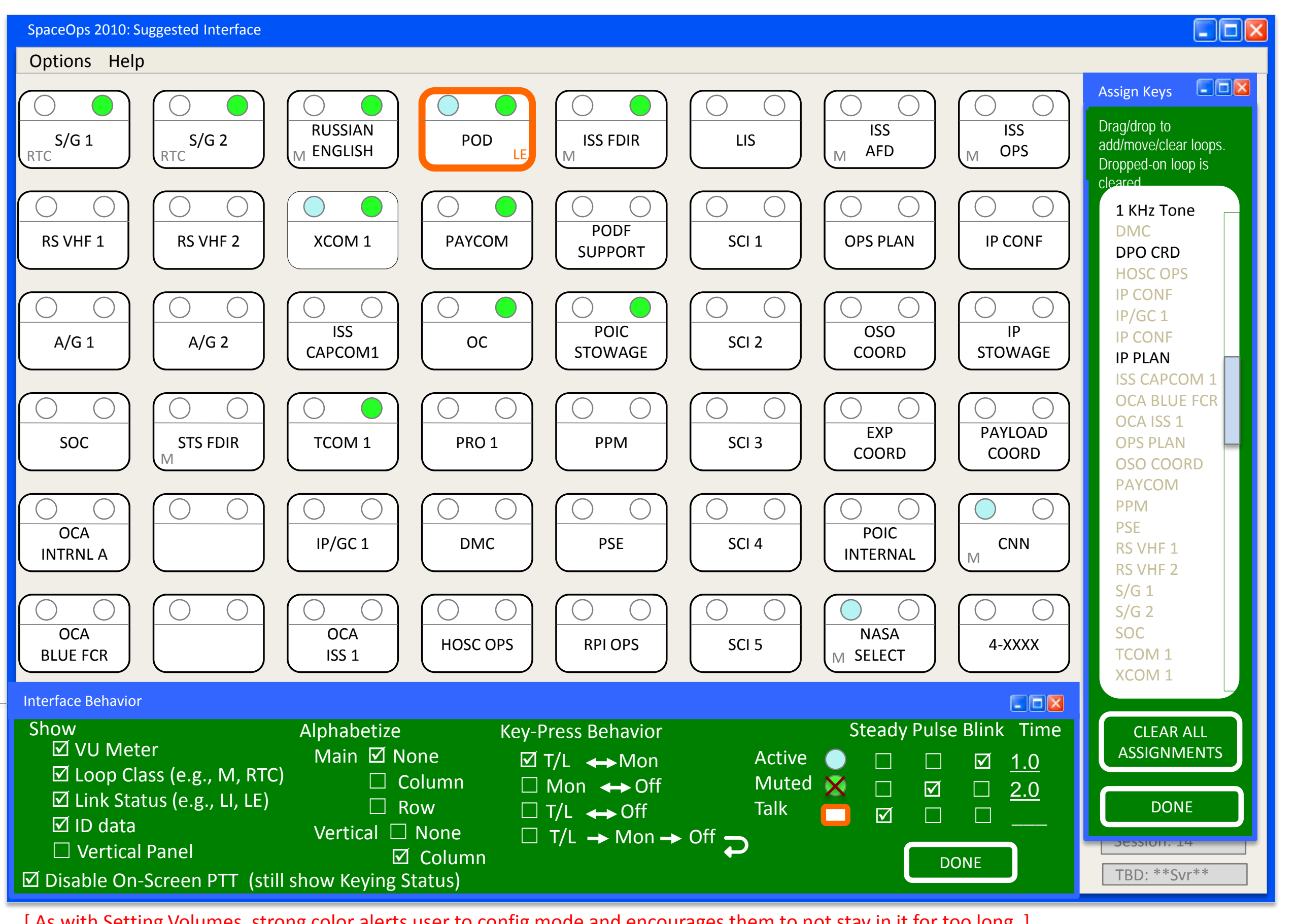

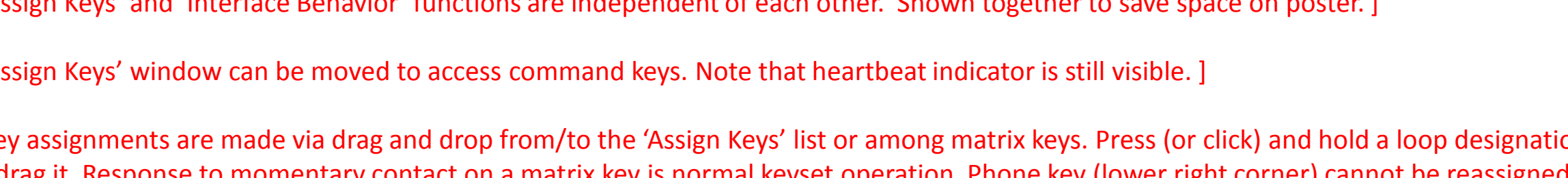

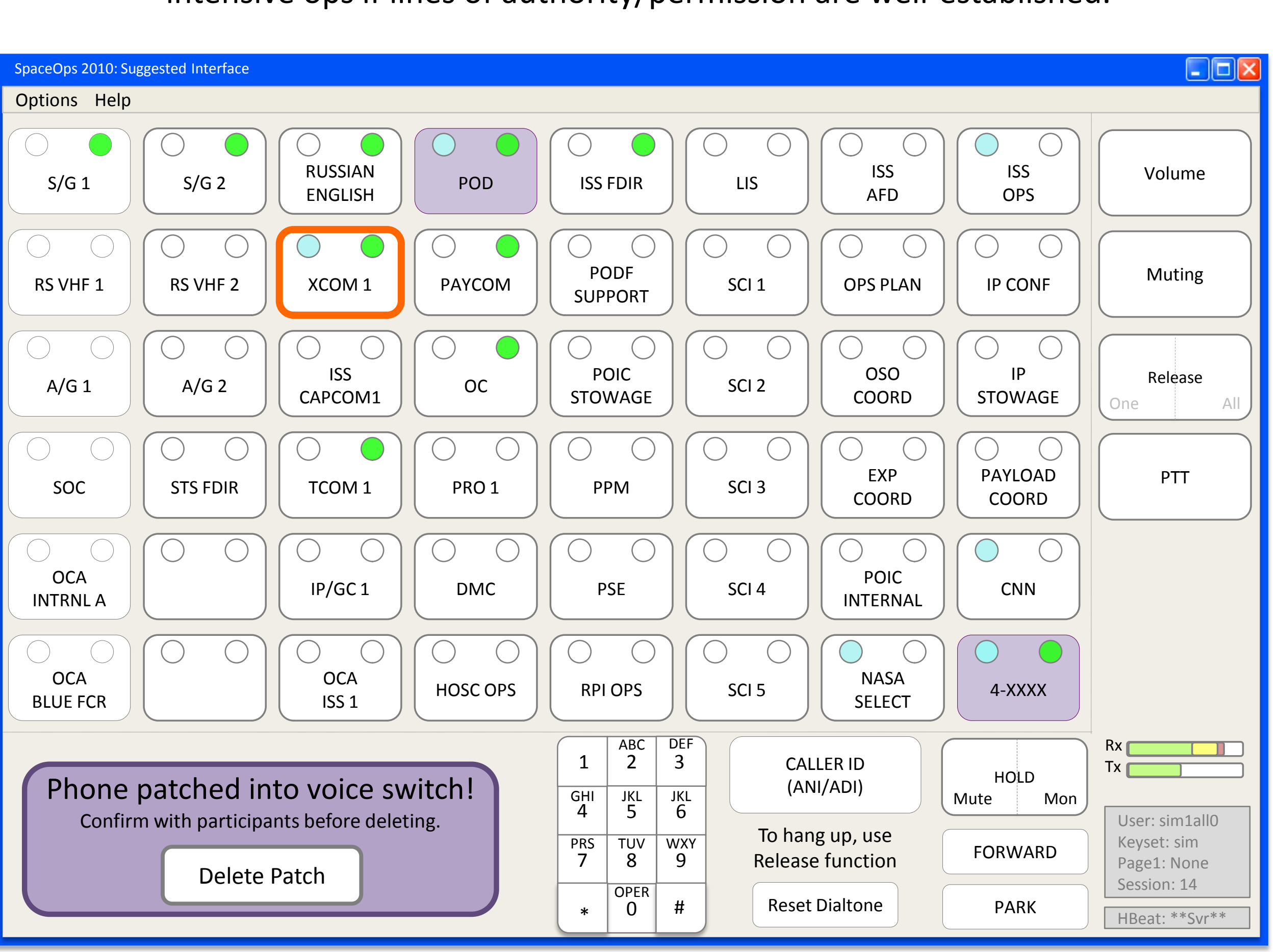

Parting Thought

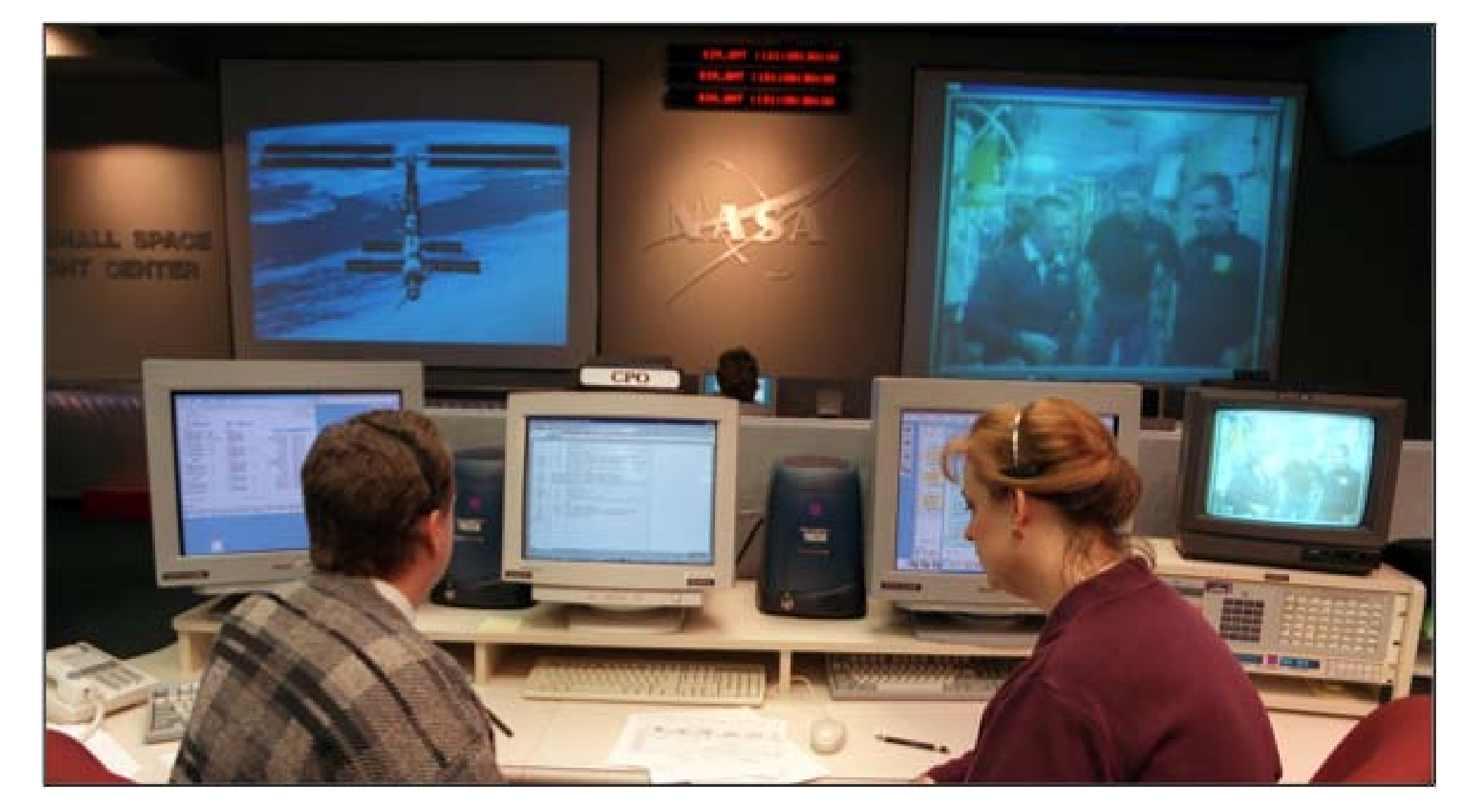

Dice communication systems are integral to operations control

Aflight controller's keyset is equivalent to a cowboy's lariat. Small irriations or in
cease to be small.

THEREFORE 